\title{
Stochastic Optimal Trajectory Generation via Multivariate Polynomial Chaos
}

\author{
Lisa Whittle * \\ European Space Agency, Keplerlaan 1, Noordwijk, The Netherlands, 2201 \\ Marco Sagliano ${ }^{\dagger}$ \\ German Aerospace Center, Robert Hooke Straße 7, Bremen, Germany, 28359
}

\begin{abstract}
This paper presents a framework that has been developed to compute stochastic optimal trajectories. This is done by transforming the initial set of stochastic ordinary differential equations into their deterministic equivalent by application of Multivariate Polynomial Chaos. Via Galerkin projection, it is possible to include stochastic information in the optimal-trajectory generation process, and to solve the corresponding Optimal Control Problem via pseudospectral methods. The resultant trajectory is less sensitive to the uncertainties included in the analysis, e.g., those present in system parameters or initial conditions. The accurate, yet computationally efficient manner in which solutions are obtained is demonstrated; a comparison with deterministic results show the benefits of the proposed approach for a linear and a non-linear problem.
\end{abstract}

\section{Nomenclature}

\begin{tabular}{ll} 
Roman & \\
$\mathbf{A}$ & Augmented matrix for state \\
$\mathbf{B}$ & Augmented matrix for control \\
$\boldsymbol{a}_{i}$ & Initial parameter PCE coefficients \\
$d$ & Number of independent random variables \\
$e_{i j k}$ & Integral of triple polynomial product \\
$F$ & Probability density function \\
$g$ & Path constraint \\
$J$ & Cost function \\
$\mathcal{L}_{2}$ & Norm space \\
$l_{b}$ & Lower bound for uniform distribution \\
$n$ & Order of polynomial expansion \\
$P$ & Order of problem \\
$t$ & Time (s) \\
$t_{0}$ & Initial time (s) \\
$t_{f}$ & Final time (s) \\
$u$ & Control \\
$u^{*}$ & Optimal Control \\
$\mathbf{u}$ & Control vector \\
$u_{b}$ & Upper bound for uniform distribution \\
$W$ & Weights from inner products \\
$x$ & States \\
$\mathbf{x}$ & State vector \\
\hline
\end{tabular}

*Young Graduate Trainee, Robotics and Future Projects Office

${ }^{\dagger}$ Research Engineer, Guidance, Navigation, and Control Department, AIAA Member 


$\begin{array}{ll}x_{0} & \text { Initial state } \\ \mathbf{x}_{0} & \text { Initial condition PCE coefficients }\end{array}$

\section{Greek}

$\begin{array}{ll}\alpha & \text { Multi index } \\ \xi & \text { Random variable } \\ \gamma & \text { Normalization factor } \\ \mu & \text { Statistical mean } \\ \mu_{a} & \text { Mean of parameter } a \\ \mu_{x} & \text { Mean of initial condition } x_{0} \\ \tau & \text { Pseudospectral domain } \\ \sigma & \text { Statistical standard deviation } \\ \sigma_{a} & \text { Standard deviation of parameter } a \\ \sigma_{x} & \text { Standard deviation of initial condition } x_{0} \\ \sigma^{2} & \text { Variance } \\ \psi & \text { Polynomial basis }\end{array}$

$\begin{array}{ll}\text { Operators } & \\ (\dot{()} & \text { First time derivative }(() / \mathrm{s}) \\ \otimes & \text { Kronecker product } \\ <\cdot> & \text { Inner product } \\ \mathbb{E}[] & \text { Expectation }\end{array}$

\section{Abbreviations}

$\begin{array}{ll}\text { AOCP } & \text { Augmented Optimal Control Problem } \\ \text { CPU } & \text { Central Processing Unit } \\ \text { DLR } & \text { Deutsches Zentrum für Luft- und Raumfahrt } \\ \text { EDL } & \text { Entry Descent and Landing } \\ \text { EKF } & \text { Extended Kalman Filter } \\ \text { fRPm } & \text { flipped Radau Pseudospectral method } \\ \text { gPC } & \text { Generalized Polynomial Chaos } \\ \text { LTI } & \text { Linear Time Invariant } \\ \text { MC } & \text { Monte Carlo } \\ \text { MPBVP } & \text { Multiple-Point Boundary-Value Problem } \\ \text { NLP } & \text { Nonlinear Programming } \\ \text { OCP } & \text { Optimal Control Problem } \\ \text { ODE } & \text { Ordinary Differential Equation } \\ \text { PC } & \text { Polynomial Chaos } \\ \text { PCE } & \text { Polynomial Chaos Expansion } \\ \text { PDF } & \text { Probability Density Function } \\ \text { PS } & \text { Pseudospectral } \\ \text { SPARTAN } & \text { Simple Pseudospectral Algorithm for Rapid Trajectory ANalysis } \\ \text { UQ } & \text { Uncertainty Quantification } \\ \text { UKF } & \text { Unscented Kalman Filtering }\end{array}$

\section{Introduction}

Extensive efforts have been devoted to the development of accurate algorithms, in order to ensure that the generated predictions are reliable and contain minimal numerical errors. Of course, in practice there are many factors that may arise which could not have been accounted for previously. This can be due 
to measurement errors, or a lack of knowledge regarding the operating conditions - perhaps measurements are infrequent, or even unattainable. For applications such as planetary landers and rovers, uncertainty is intrinsic. Whether it is manifested in the landing location, or as a consequence of mismatch in atmospheric conditions, failure to acknowledge this could be detrimental to the mission. Therefore, appropriate treatment of these uncertainties must be integrated within the computational process, so that it becomes possible to fully understand the impact of errors, or uncertainty in parameter values, initial and boundary conditions. Consequently, the field of Uncertainty Quantification (UQ) has become prominent in recent years, and it has since become possible to investigate the effect of such errors in measurements and/or modeling. One of the most promising methods for UQ is Generalized Polynomial Chaos (gPC). Based on the original theory of Norbert Wiener in 1938 regarding Hermite Homogenous Chaos, ${ }^{1}$ it incorporates orthogonal polynomials in order to express the random quantities. Through exploitation of their inherent characteristics, the stochastic quantities are transformed into a set of augmented deterministic equations. This generalization was developed by Ghanem and Spanos and applied to many practical problems, ${ }^{2}$ as a framework to overcome some of the prior convergence issues when used in application to non-Gaussian problems. This is due to the fact that full convergence may only be achieved if the appropriate orthogonal polynomials are selected, based on the distributions of the random parameter. ${ }^{3}$ Upon selection of the appropriate polynomial basis, it is necessary to solve the problem using one of two methods: Stochastic Collocation or Galerkin Projection. The Galerkin method essentially minimizes the error of the finite-order gPC expansion using projection principles, resulting in a set of coupled deterministic equations that can then be solved using a numerical solver, such as Euler or Runge-Kutta. A benefit of the latter method is it requires far fewer equations than Stochastic Collocation in order to achieve polynomial exactness. ${ }^{4}$ However, implementation is not as straightforward, as the Galerkin system must be derived; a challenging process for complex, non-linear applications. For this reason, Galerkin techniques are not as widely adopted, despite the fact they offer accurate solutions for multi-dimensional problems, and do so using a minimal number of equations - potentially leading to clear improvements in terms of computational efficiency. ${ }^{5}$

Evidently there are many benefits posed by application of gPC methods, however, their application to controller design has only recently become apparent. Development of gPC applications in the context of robust control was most notably due to Nagy and Braatz, who demonstrated the influence of parametric uncertainty upon non-linear systems in industrial applications such as batch crystallization. ${ }^{6}$ This served as a verification and validation technique, however, stability was not analyzed. Application of gPC for stability analysis was first demonstrated for a simple bi-linear system by Hover and Triantafyllou. ${ }^{7}$ Combination of robust control algorithms and stochastic control methods makes it possible to first understand how random uncertainties impact the state trajectories of the system in question. Secondly, it results in a less conservative controller design, thus ensuring the most effective performance. The stability margin of the controller is therefore designed accounting for this uncertainty, which results in a probabilistic robust control framework.

It is important to understand how these stochastic quantities evolve and manifest within the system. The first to demonstrate a novel uncertainty propagation framework for hypersonic flight dynamics using gPC was Prabhakar et al in 2010. ${ }^{8}$ This concerned a Mars Entry, Descent and Landing (EDL) problem, comprising of structured uncertainties in both the initial conditions and the state parameters. Later that year, Dutta and Bhattacharya expanded this work by means of Bayesian estimations of the a priori Probability Density Function (PDF) of the random process, for a non-linear problem. ${ }^{9}$ The combination of Bayesian estimator and $\mathrm{gPC}$ framework performed very well for the non-linear, hypersonic problem, in comparison to linear estimators. In particular, it was shown that the Extended Kalman Filter (EKF) performs poorly for non-Gaussian parameter uncertainty. At this point it is important to specify the cost functionals that are presented within the gPC stochastic framework. Specifically, the aim is to deduce trajectories that result in minimum expectation and/or minimum variance. This was covered by Fisher and Bhattacharya in 2011, when they demonstrated that the derived stochastic cost was indeed equivalent to the standard quadratic cost function. ${ }^{10}$ This was also shown by Xiong et al, who used these optimization principles in application to the Hypersensitive and Van der Pol problems. ${ }^{11}$ These examples were univariate stochastic problems (i.e., with uncertainty in the parameter or initial condition). Multivariate, mixed-distribution problems have also been covered by Xiong, although not in the context of optimization. ${ }^{12}$

High accuracy is demonstrated by gPC, and with the added benefit of reduction in computational cost when compared to methods such as Monte Carlo (MC). MC is very popular due to its relative simplicity, and is usually the first method employed when analyzing such stochastic problems. Unfortunately, the pure repetition required in order to obtain deterministic solutions places a very significant computational 
burden, as the mean value generally converges at a rate inverse to the number of samples. Furthermore, it is not computationally scalable and can suffer statistical inconsistencies. Other methods used in uncertainty propagation include: Markov Chain MC, Bayesian Estimators and Unscented Kalman Filtering (UKF). The elegant transformation from stochastic to deterministic problem offered by gPC methodology is promising due to its competitive accuracy and efficiency, and is particularly attractive in the context of stochastic trajectory optimization. In fact, this transformation makes the gPC framework compatible with any transcription method and optimization process. It is therefore possible to use it to generate an Augmented Optimal Control Problem (AOCP), which is, from the formal point of view, a standard Optimal Control Problem (OCP).

In an OCP we are interested to determine the controls and states that optimize a performance cost, with respect to dynamic and/or algebraic constraints. These are in the form of Ordinary Differential Equations (ODEs), boundary conditions, and path constraints. The problem, in general Bolza form, is solved using one of two classes of methods: indirect or direct. Indirect methods utilize Pontryagin's principle to formulate the problem in terms of minimization of Hamiltonian, whilst direct methods involve transcription of the OCP into a finite dimensional, Non-Linear Programming (NLP) problem. ${ }^{13}$ Given their ease of use when compared to indirect methods, direct methods are favorable and widely adopted nowadays. Among the several tools employing direct methods there is SPARTAN, ${ }^{14,15}$ a MATLAB tool developed at the German Aerospace Center (DLR), which utilizes pseudospectral (PS) methods in order to solve the OCP. More specifically, pseudospectral methods offer full capability for uncertainty propagation using the relevant quadrature (dependent on the distribution of the random quantity) and then SPARTAN solves the augmented stochastic system in order to deduce the optimal solution to the corresponding AOCP. This paper therefore extends prior work within the field by combining multi-dimensional, mixed-distribution, uncertainty propagation using gPC and the obtainment of the optimized control solution. Thus, this framework enables calculation of far more robust trajectories in the presence of structured uncertainties.

The remainder of this paper is organized as follows. In Sec. II a brief overview on PS methods is given. The proposed stochastic trajectory optimization procedure is explained in Sec. III. The results obtained for two different examples using this framework can be found in Sec. IV. Finally, some conclusions about this work, are drawn in Sec. V, together with the possible directions for the future work.

\section{Overview on Pseudospectral Methods}

\section{A. Optimal Control Problem}

There are several approaches for the generation of reference trajectories. Some methods exploit the structure of the specific problem to be dealt with. Often, they require simplifications in order to make the problem mathematically tractable, and therefore generate solutions valid under given hypotheses. A different approach, which is gaining popularity, and benefits from the development of the computational capabilities of modern CPUs, is the representation of the trajectory generation problem as an Optimal Control Problem (OCP). This means that solutions which minimize (or maximize) a given criterion are sought, that at the same time satisfy several constraints, which can be differential (i.e., the equations of motion of a spacecraft) and/or algebraic (e.g., the maximum heat-flux that a vehicle can tolerate during the atmospheric entry). The standard form for representing OCPs is the so-called Bolza problem. Given a state vector $\mathbf{x}(t) \in \mathbb{R}^{n_{s}}$, a control vector $\mathbf{u}(t) \in \mathbb{R}^{n_{c}}$, the scalar functions $\Phi(t, \mathbf{x}, \mathbf{u})$ and $\Psi(t, \mathbf{x}, \mathbf{u})$, and the vector $\mathbf{g}(t, \mathbf{x}, \mathbf{u}) \in \mathbb{R}^{n_{g}}$, we can formulate the problem as follows:

$$
\operatorname{minimize} \quad J=\Phi\left[t_{f}, \mathbf{x}\left(t_{f}\right), \mathbf{u}\left(t_{f}\right)\right]+\int_{t_{0}}^{t_{f}} \Psi[\mathbf{x}(t), \mathbf{u}(t)] d t
$$

subject to the differential equations

$$
\dot{\mathbf{x}}=\mathbf{f}(t, \mathbf{x}, \mathbf{u})
$$

and to the path constraints

$$
\mathbf{g}_{L} \leq \mathbf{g}(t, \mathbf{x}, \mathbf{u}) \leq \mathbf{g}_{U}
$$

The first term in the cost function of Eq. (1) takes the name of Mayer term, and represents punctual constraints (e.g., the minimization of a distance according to a given metric), while the argument of the integral is called the Lagrange term and is used to maximize or minimize variables over the entire mission 
(e.g., the heat load obtained by integrating the heat-flux over time). The inequalities in Eq. (3) are intended as component-wise. Note that although not specifically expressed, we always refer to autonomous systems of differential equations. Therefore the time dependency in Eq. (2) is never explicit. Moreover, since physical systems are dealt with, the problem usually has bounded states and controls; that is, $\mathbf{x}(t)$ and $\mathbf{u}(t)$ are compact in $\mathbb{R}^{n_{s}}$ and $\mathbb{R}^{n_{c}}$, respectively:

$$
\begin{aligned}
& \mathbf{x}_{L} \leq \mathbf{x}(t) \leq \mathbf{x}_{U} \\
& \mathbf{u}_{L} \leq \mathbf{u}(t) \leq \mathbf{u}_{U}
\end{aligned}
$$

Moreover, initial and final conditions might be constrained as well.

$$
\begin{aligned}
& \mathbf{x}_{0}=\mathbf{x}\left(t_{0}\right) \\
& \mathbf{x}_{f}=\mathbf{x}\left(t_{f}\right)
\end{aligned}
$$

Eqs. (1)-(7) represent a generic continuous OCP. In the next section, it is demonstrated exactly how this type of problem can be transcribed by using pseudospectral (PS) methods.

\section{B. Pseudospectral methods}

Numerical methods for solving OCPs are divided into two major classes, namely, indirect methods and direct methods. Indirect methods are based on the Pontryagin's Maximum Principle, which leads to a MultiplePoint Boundary-Value Problem (MPBVP). Direct methods, instead, consist of the proper discretization of the OCP (or transcription), which results in a finite-dimensional NLP problem. Pseudospectral (PS) methods represent a particular area of interest in the frame of the wider class of direct methods. Examples of tools implementing PS methods include DIDO, ${ }^{16}$ GPOPS $^{17}$ and SPARTAN. ${ }^{14,15}$ For these methods, the following properties are valid:

- "Spectral" (i.e., quasi-exponential) convergence of the NLP solution to the OCP solution when the number of nodes employed is increased (and the problem is smooth)

- Runge phenomenon is avoided

- Sparse structure of the associated NLP problem

- Mapping between the discrete costates of the associated NLP and the continuous costates of the OCP in virtue of the Pseudospectral Covector Mapping Theorem. ${ }^{18}$

The transcription process does not only involve the choice of the discrete nodes, but also determines the discrete differential and integral operators needed to solve the associated OCP. Therefore, transcription is a more general process than discretization. The minimum fundamental steps of a transcription are the following:

- Domain discretization

- Discrete to continuous conversion of states and/or controls

- Characterization of differential and integral operators

Among the families of pseudospectral (PS) methods, a specific one was considered for this work: the flipped Radau Pseudospectral method (or fRPm). It is worth noting that this is not the only possible choice, as other sets of nodes, like Gauss or Lobatto exist. ${ }^{19}$ The reason behind this choice is that the fRPm allows for a natural and straightforward definition of the initial conditions of the problem, and demonstrates a smoother convergence of the costates with respect to other methods. ${ }^{19}$

\section{Flipped Radau Pseudospectral method}

Flipped Radau Pseudospectral method is an asymmetric PS method, whose nodes are the roots of the flipped Legendre-Radau polynomial, defined as the combination of the Legendre polynomial of order $n$ and $n-1$ with coefficient equal to 1 and -1 , respectively.

$$
R_{n}(\tau)=\widetilde{L}_{n}(\tau)-\widetilde{L}_{n-1}(\tau) \quad \tau \in[-1,1]
$$


An example of roots associated with the Legendre-Radau polynomial of order 10 is depicted in Fig. 1(a), together with the corresponding polynomial.

Remark 1 Note that the $R_{n}(-1)$ is not a root of the underlying polynomial, therefore it is not a collocation point, although it is required for the evaluation of the polynomial. This is due to the fact that only over the left-open, right-closed interval $(-1,+1]$ these polynomials are orthogonal.

This discrete representation of the domain is useful when reconstructing continuous approximations of the functions $x(t)$ as:

$$
x(t) \cong \sum_{i=0}^{n} X_{i} P_{i}(t), \quad P_{i}(t)=\prod_{\substack{k=0 \\ k \neq i}}^{n} \frac{t-t_{k}}{t_{i}-t_{k}}
$$

A property of Radau PS methods is that only one of the two extremal points is collocated. This difference will affect the differential operators which will be introduced in the next section.

An example of the approximation obtained via Eq. (9) is depicted in Fig. 1(b), where the function $1 /\left(1+25 \tau^{2}\right)$ is reconstructed by using 25 nodes. It is possible to see that the original function is approximated very well with this set of discrete nodes.

Remark 2 Note that the approximation becomes more accurate when the number of nodes is increased. This is the opposite behavior observed when uniform distributions of nodes, which suffer from the aforementioned Runge Phenomenon, are employed. Once that the domain has been discretized, and the discrete-to-continuous conversion of states has

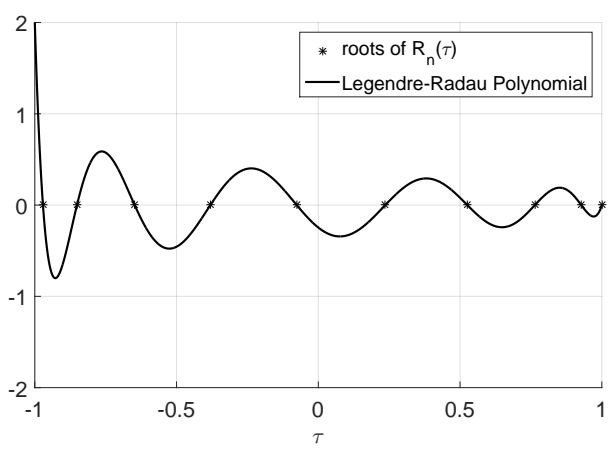

(a) fRPm discrete domain.

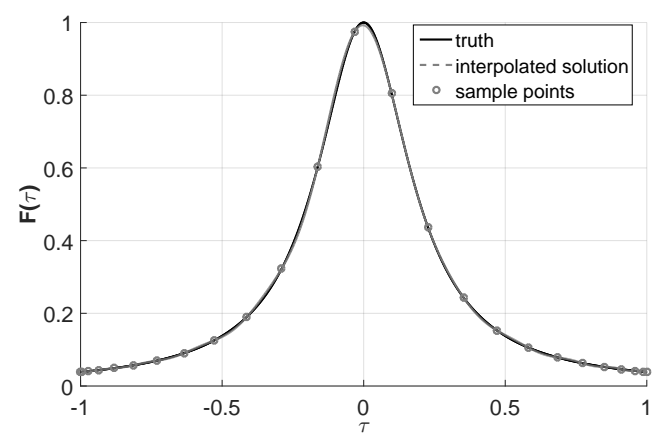

(b) fRPm continuous approximation of a function.

Figure 1: Transcription steps with fRPm: (a) domain discretization, (b) continuous reconstruction of functions.
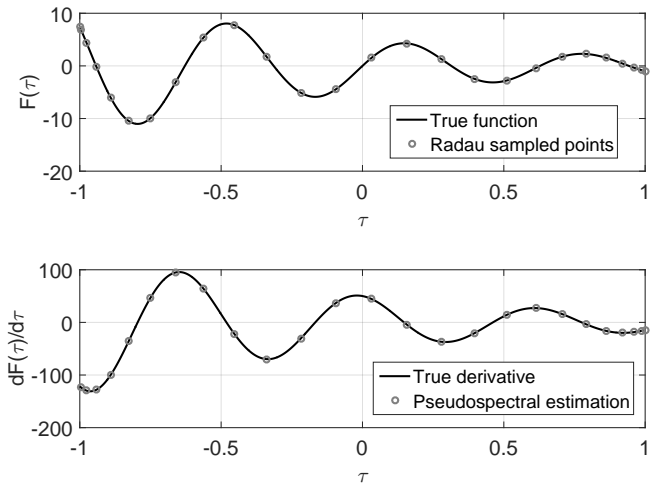

(a) fRPm differential operator example.
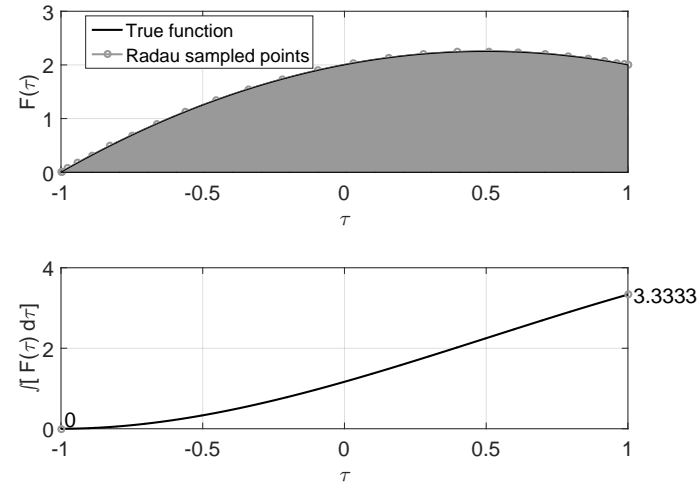

(b) fRPm integral operator example.

Figure 2: Transcription steps with fRPm: (a) definition of differential operators, (b) application of integral operator.

been defined, the corresponding differential operator needs to be defined. This is required for the proper representation of the left-hand side of Eq. (2). The differential operator will be in the form:

$$
\dot{\mathbf{X}} \cong \mathbf{D} \cdot \mathbf{X}
$$


and the dynamics defined in Eq. (2) will be replaced by:

$$
\mathbf{D} \cdot \mathbf{X}=\frac{t_{f}-t_{0}}{2} \mathbf{f}(t, \mathbf{X}, \mathbf{U})
$$

where $t_{0}$ and $t_{f}$ are the initial and final time, and the term $\frac{t_{f}-t_{0}}{2}$ is a scale factor related to the transformation between the physical time domain $t$, and the pseudospectral time domain $\tau \in[-1,1]$, given by the following affine transformations:

$$
\begin{aligned}
& t=\frac{t_{f}-t_{0}}{2} \tau+\frac{t_{f}+t_{0}}{2} \\
& \tau=\frac{2}{t_{f}-t_{0}} t-\frac{t_{f}+t_{0}}{t_{f}-t_{0}}
\end{aligned}
$$

The matrix $\mathbf{D}$ has dimensions $[n \times(n+1)]$. Again, this is due to the fact that the states are defined for $n+1$ discrete points, while the controls $\mathbf{U}$ and the derivatives of the states $\mathbf{f}(t, \mathbf{X}, \mathbf{U})$ are defined in the $n$ collocation points. This means that the initial state $\mathbf{X}_{0}$ is an input and not an output of the optimization in the fRPm, and it is thus assumed to be known. If we look at Eq. (9) and we take the derivative w.r.t. time, we get:

$$
\dot{\mathbf{x}}(t) \cong \frac{d}{d t} \sum_{i=0}^{n} \mathbf{X}_{\mathbf{i}} P_{i}(t)=\sum_{i=0}^{n} \mathbf{X}_{\mathbf{i}} \frac{d}{d t} P_{i}(t)
$$

as the nodal points are time-independent. These derivatives can be efficiently computed with the Barycentric Lagrange Interpolation. ${ }^{20}$ An example of the differential operator for the method is depicted in Fig. 2(a), where $\mathbf{D}$ is used to approximate the derivative of the continuous test function $F(\tau)=A e^{-\tau} \sin (\omega \tau)$, $(A=5$, $\omega=10)$ sampled in 25 collocation nodes. It can be seen that the polynomial approximations fit the derivatives very well.

In addition to the differential operator, we need an integral operator. This operator is required as the cost function in Eq. (1) may contain the Lagrange term, which needs proper discretization. In that case, the Gauss quadrature formula is used. ${ }^{21}$ For the fRPm, the approach consists of replacing the continuous integral with the discrete sum given by:

$$
\int_{t_{0}}^{t_{f}} \Psi[t, \mathbf{x}(t), \mathbf{u}(t)] d t=\frac{t_{f}-t_{0}}{2} \sum_{i=1}^{n} w_{i} \Psi\left[\mathbf{X}_{i}, \mathbf{U}_{i}\right]
$$

It can be shown that Eq. (15) yields exact results for polynomials of order at most equal to $2 n-2 .{ }^{19}$ Once again, the presence of the term $\frac{t_{f}-t_{0}}{2}$ is a consequence of the mapping between pseudospectral and physical time domains described in Eq. (12) and (13). The weights, $w_{j}$, can be computed as:

$$
\begin{gathered}
w=f \operatorname{lip}(\tilde{w}) \\
\tilde{w}_{j}= \begin{cases}\frac{2}{n^{2}}, & j=1 \\
\frac{\left(1-\tau_{j}\right)}{n^{2} \tilde{L}_{n}\left(\tau_{j}\right)^{2}}, & j=[2, \ldots n]\end{cases}
\end{gathered}
$$

where the operator flip multiplies the input by a factor equal to -1 , and sorts the results in increasing order. To give a practical example, the integral of the test function $F(\tau)=2 \tau+2-\tau^{2}$ has been computed. Results are then compared with the analytical integral, and with the trapezoidal rule (Fig. 2(b)) applied using the same nodes. Numerically, we get exactly the analytical result, that is 3.3333 when fRPm is employed, while the application of the trapezoidal rule gives 3.3298; confirming the validity of the quadrature formula applied to the fRPm points. Note that when $n$ uniformly distributed nodes are used, the trapezoidal rule gives better results (3.3310), but is still inferior to the pseudospectral ones.

Having given an overview of PS methods and the definition of differential and integral operators, it is now necessary to detail the process of stochastic trajectory optimization. 


\section{Proposed Method for Stochastic Trajectory Optimization}

In this section the proposed stochastic trajectory optimization method is described. First, the concept of Polynomial Chaos (PC) for uncertainty propagation is briefly summarized. Secondly, the Galerkin projection method used to obtain the augmented system of differential equation is detailed. This projection leads to the proper definition of the underlying Augmented Optimal Control Problem (AOCP), which is ultimately solved with SPARTAN.

\section{A. Uncertainty Propagation}

In general terms, a dynamic system may be represented by means of Ordinary Differential Equations (ODEs), which can then be solved by a suitable numerical integration scheme (Runge Kutta, for instance). Let us consider a simple linear case:

$$
\text { Deterministic dynamics }\left\{\begin{array}{l}
\dot{x}(t)=a x(t) \\
x_{0}=x\left(t_{0}\right)
\end{array} \quad t \in\left[t_{0}, t_{f}\right]\right.
$$

where $\mathbf{x} \in \mathbb{R}$ is the state, and $a \in \mathbb{R}$ represents the system parameter. The initial state is denoted by $x_{0}$ and consequently integrated over the time span $\left[t_{0}, t_{f}\right]$. In the deterministic case (i.e., no uncertainties are present in the initial condition or parameter), this governing equation leads to a deterministic solution; however, in the presence of uncertainties Eq. (18) becomes a stochastic differential equation, which has a stochastic solution $x(t)$. A way to deal with stochastic systems is represented by the application of Polynomial Chaos Expansion (PCE). If we consider that stochastic quantities arise from both the parameter, $a$, and the initial condition, $x_{0}$, then both the state $x(t)$ and the parameter $a$ can be represented by the corresponding PCE models:

$$
\text { PCE models }\left\{\begin{aligned}
x(t) & =\sum_{j=0}^{\infty} x_{j}(t) \psi_{j}(\xi) \\
a(t) & =\sum_{i=0}^{\infty} a_{i} \psi_{i}(\xi)
\end{aligned}\right.
$$

The respective stochastic variables are represented by $\xi=\left[\xi_{1}, \ldots, \xi_{d}\right]$, where $d$ is the number of uncertainties in the system. This infinite-dimensional expansion can be truncated at a suitable order, $P$, according to the work of Cameron and Martin, who stated full convergence in the $\mathcal{L}_{2}$ sense may be achieved upon appropriate orthogonal polynomial selection. ${ }^{22}$ This truncation is performed in consideration of both numerical accuracy and computational cost. The correct choice is dependent on the weighting of the polynomial being compatible with the Probability Distribution Function (PDF), as highlighted in Tab. 1.

Table 1: Orthogonal polynomial selection based on PDF of stochastic quantity.

\begin{tabular}{cccc}
\hline \hline Distribution & Corresponding Polynomial & Interval & Weighting \\
\hline \hline Gaussian & Hermite & $(-\infty, \infty)$ & $e^{-x^{2} / 2}$ \\
\hline Uniform & Legendre & {$[-1,1]$} & $\frac{1}{2}$ \\
\hline \hline
\end{tabular}

Consequently, the PCE models given by Eq. (19) are reduced to the $P^{\text {th }}$ order approximation, and then substituted into the original ODE (Eq. (18)).

Hence the stochastic linear system is:

$$
\sum_{k=0}^{P} \dot{x}_{k}(t) \psi_{k}(\xi)=\sum_{i=0}^{P} \sum_{j=0}^{P} a_{i} x_{j}(t) \psi_{i}(\xi) \psi_{j}(\xi)
$$

which represents a set of $P+1$ coupled differential equations. The extension to larger dimensions is obtained by applying the expansion to each of the terms involved in the original set of differential equations, and leads 
to the overall dimension of the problem, which is deduced using the following relationship,

$$
P+1=\frac{(n+d) !}{n ! d !}
$$

where $n$ is the highest degree of polynomial used within the expansion, and the number of independent random variables is given by $d$. Further details regarding the multivariate expansion can be found in Appendix B. Therefore, $P+1$ determines the number of PCE coefficients resulting from the expansion, which are used in order to transform the stochastic model into the corresponding deterministic augmented system. Unfortunately, the dimension of the problem will grow very rapidly with increasing $n$ and/or $d$, thus creating significant computational burden. However, the curse of dimensionality is partially mitigated by

1. Choosing the set of basis functions corresponding to the uncertainty (for instance, for normally distributed variables an expansion of $n=2$ is perfectly sufficient ${ }^{4}$ ).

2. Applying efficient multivariate integration techniques, based for instance on pseudospectral hyperquadrature rules.

\section{B. Galerkin Projection}

The next step is to perform the Galerkin projection, which will result in a set of $P+1$ deterministic equations. This coupled system is given by the following relationship, where $<\cdot>$ represents the inner product.

$$
\dot{x}_{k}(t)=\frac{1}{<\psi_{k}^{2}(\xi)>}<\sum_{j=0}^{P} x_{j}(t) \psi_{j}(\xi), \sum_{i=0}^{P} a_{i} \psi_{i}(\xi), \psi_{k}(\xi)>\quad k \in[0, \ldots, P]
$$

The inner product of the orthogonal polynomials is the integral of the product of univariate or multivariate polynomial bases, $\psi_{k}(\xi)$, and the probability distribution, $F(\xi)$.

$$
<\psi_{i}(\xi), \psi_{j}(\xi)>=\int_{a}^{b} \psi_{i}(\xi) \psi_{j}(\xi) F(\xi) d \xi
$$

The limits $a$ and $b$ are the integral bounds for the respective distribution (see Tab. 1). Note that in the multivariate case, the PDF is then simply the product of the marginal distributions for each random variable, if they are mutually independent. Thus, even in the case of mixed distribution problems, the system characteristics can be captured well.

The initial PCE coefficients for the parameter PCE are given by $\boldsymbol{a}_{i}$, which consist of the mean, $\mu_{a}$, and standard deviation, $\sigma_{a}$. For problems which contain only parameter uncertainty, the properties of the random parameter are placed in the first and second entries, respectively and all other values are zero. The dimension is given by $\boldsymbol{a}_{i} \in \mathbb{R}^{[1 \times P+1]}$ (i.e. $\boldsymbol{a}_{i}=\left[\mu_{a}, \sigma_{a}, 0, \ldots, 0\right]$ ). However, in the multi-dimensional case it is slightly different (which will be described fully as follows). If we now denote the integral of the triple product given by Eq. (23), as $e_{i j k}$, and the normalization factor (as it is also known), as $\gamma_{k}$, then the augmented system can be written in such a manner:

$$
\dot{x}_{k}(t)=\frac{1}{\gamma_{k}} \sum_{i=0}^{P} \sum_{j=0}^{P} a_{i} x_{j}(t) e_{i j k}
$$

where,

$$
e_{i j k}=\mathbb{E}\left[\psi_{i}(\xi) \psi_{j}(\xi) \psi_{k}(\xi)\right]
$$

and

$$
k \in[0,1, \ldots, P], \quad \gamma_{k}=<\psi_{k}(\xi), \psi_{k}(\xi)>
$$

Note that for the one-dimensional case, there exists an analytical solution ${ }^{23}$ for the integral of the triple product, $e_{i j k}$, and the normalization factor, $\gamma_{k}$, for Hermite and Legendre polynomials, i.e., Gaussian and uniformly distributed random quantities $\xi$. Specifically, we have: 
Hermite polynomials:

$$
e_{i j k}=\left\{\begin{array}{l}
\frac{i ! j ! k !}{(s-i) !(s-j) !(s-k) !}, \quad \gamma_{k}=k ! \quad \text { if Eq. (29) holds } \\
0 \quad \text { otherwise }
\end{array}\right.
$$

Legendre polynomials:

$$
e_{i j k}=\left\{\begin{array}{l}
\left((-1)^{s} \sqrt{\frac{(2 s-2 i) !(2 s-2 j) !(2 s-2 k) !}{(2 s+1) !} \frac{s !}{((s-i) !(s-j) !(s-k) !}}^{2}, \quad \gamma_{k}=\frac{1}{2 k+1} \quad\right. \text { if Eq. (29) holds } \\
0 \quad \text { otherwise }
\end{array}\right.
$$

The orthogonality conditions are given by:

$$
s \geq i, j, k \text { and } 2 s=i+j+k \text { is even }
$$

The solutions can of course also be obtained using the quadrature methods. If only one uncertainty is introduced, then the order of these polynomials is simply given by the indices $i, j$ and $k$. However, in the multivariate case, the polynomial bases are built using a Graded Lexicographic indexing method. ${ }^{4}$ This indexing method involves generating an array of indices of dimension $[(P+1) \times d]$ and essentially generates every combination of integers whose sum equals the order of polynomial expansion, $n$. For example, consider a problem with 2 uncertain quantities $(d=2)$, and with a third order PCE $(n=3)$. Using this method, the polynomial bases are built as follows, where the subscript following $\psi$ denotes the order of polynomial and the subscript following $\xi$ corresponds to the appropriate random variable (Tab. 2). The integral of the triple product, $e_{i j k}$, for the multivariate case is therefore found using hyperquadrature techniques (i.e., quadrature

\begin{tabular}{|c|c|c|c|}
\hline$|i|$ & $\alpha$ & Single index k & Polynomial basis \\
\hline 0 & 00 & 0 & $\psi_{0}\left(\xi_{1}\right) \psi_{0}\left(\xi_{2}\right)$ \\
\hline \multirow[t]{2}{*}{1} & 10 & 1 & $\psi_{1}\left(\xi_{1}\right) \psi_{0}\left(\xi_{2}\right)$ \\
\hline & 01 & 2 & $\psi_{0}\left(\xi_{1}\right) \psi_{1}\left(\xi_{2}\right)$ \\
\hline \multirow[t]{3}{*}{2} & 20 & 3 & $\psi_{2}\left(\xi_{1}\right) \psi_{0}\left(\xi_{2}\right)$ \\
\hline & 11 & 4 & $\psi_{1}\left(\xi_{1}\right) \psi_{1}\left(\xi_{2}\right)$ \\
\hline & 02 & 5 & $\psi_{0}\left(\xi_{1}\right) \psi_{2}\left(\xi_{2}\right)$ \\
\hline \multirow[t]{4}{*}{3} & 30 & 6 & $\psi_{3}\left(\xi_{1}\right) \psi_{0}\left(\xi_{2}\right)$ \\
\hline & 21 & 7 & $\psi_{2}\left(\xi_{1}\right) \psi_{1}\left(\xi_{2}\right)$ \\
\hline & 12 & 8 & $\psi_{1}\left(\xi_{1}\right) \psi_{2}\left(\xi_{2}\right)$ \\
\hline & 03 & 9 & $\psi_{0}\left(\xi_{1}\right) \psi_{3}\left(\xi_{2}\right)$ \\
\hline
\end{tabular}
for higher dimensions).

Table 2: Multi-Index Method for $n=3, d=2$.

Now the integral, $e_{i j k}$, is also generated using the relation given by Eq. (25), however, the single integer is now of dimension $d$, corresponding to the number of independent stochastic variables. The multi-index, $\alpha$, is also intrinsically linked to the definition of the initial PCE coefficients $\boldsymbol{a}_{i}$ and $\mathbf{x}_{0}$. If we first consider the parameter $\boldsymbol{a}_{i}$, the multi-index infact dictates the positioning of the distribution parameters $\left(\mu_{a}\right.$ and $\left.\sigma_{a}\right)$. Taking the example given for $n=3, d=2$, the initial PCE coefficients are then: $\boldsymbol{a}_{i}=\left[\mu_{a}, 0, \sigma_{a}, 0,0,0\right]$. The corresponding polynomial bases will define a tensor product (also called Kronecker product, denoted by $\otimes)$ given in the fourth column of Tab. 2. The normalization factor, $\gamma_{k}$, will be defined accordingly as the product of the univariate $\gamma_{k}$ :

Hermite polynomials:

$$
\gamma_{k}=\prod_{i=0}^{d} \alpha_{i} !
$$


Legendre polynomials:

$$
\gamma_{k}=\prod_{i=0}^{d} \frac{1}{2 \alpha_{i}+1}
$$

Remark 3 Two classes of Hermite polynomials exist: physicists' and probabilists'. Here the latter is used. In the case of Legendre polynomials, there are also two variations: normal and shifted. They each have different normalization factors and thus, care must be taken to ensure full compatibility. In this work we will always refer to the normal Legendre polynomials.

In the case of mixed distributions, the normalization is simply the product of the respective normalization factors, $\gamma_{k}$. By solving these integrals given by Eqs. (23) and (26), it is possible to compute an augmented matrix, A, that is used in the new system of equations. For example, let us consider the linear case as before, but now with a control input, $u$ subject to the parameter, $b$ (i.e., a continuous-time linear system):

$$
\text { LTI system }\left\{\begin{array}{l}
\dot{x}(t)=a x(t)+b u(t) \\
x(0)=x_{0}
\end{array}\right.
$$

We then assume that there is uncertainty in the variable $x$ (i.e., resulting from parameter, $a$, and/or initial condition, $x_{0}$ ), whilst the control, $u$ is deterministic ( $b$ is constant). The stochastic system representing Eq. (32) is given by the augmented linear system.

$$
\dot{\mathbf{x}}(t)=\mathbf{A x}(t)+\mathbf{B u}(t)
$$

where the state vector is $\mathbf{x}=\left[x_{0}, x_{1}, \ldots, x_{p}\right]^{T}$ and the control vector is defined as $\mathbf{u}=\left[u_{0}, u_{1}, \ldots, u_{p}\right]^{T}$. The state transformation matrix $\mathbf{A}$ is built corresponding to the $k^{\text {th }}$ row and $j^{\text {th }}$ column and will be of dimension $\mathbf{A} \in \mathbb{R}^{n_{s}(P+1) \times n_{s}(P+1)}:$

$$
A_{k, j}=\frac{1}{\gamma_{k}} \sum_{i=0}^{P} \sum_{j=0}^{P} a_{i} e_{i j k} \quad k \in[0, \ldots, P]
$$

Since we assumed a deterministic control parameter, the corresponding transformation matrix is simply $\mathbf{B}=[1,0, \ldots, 0]^{T}$ and is of dimension $\mathbf{B} \in \mathbb{R}^{n_{s}(P+1) \times n_{c}}$.

The system of Eq. (34) can be propagated in order to obtain the PCE coefficients (as now given by each of the components of $\mathbf{x}$ ). The initial values of $\mathbf{x}$ used for the propagation are $\mathbf{x}_{0}$, which correspond to the first PCE coefficients of the initial condition. For instance, if there is uncertainty in the initial conditions, then $\mathbf{x}_{0}=\left[\mu_{x}, \sigma_{x}, 0, \ldots, 0\right]^{T}$. If uncertainty is only in the parameter, $a$, then $\mathbf{x}_{0}$ will only consist of $\mu_{x}$ as a non-zero term. This further conveys how the multi-index, $\alpha$, influences the initial conditions. Note that the initial condition uncertainty is always assigned before that of the parameter.

Now the generation of the multivariate PCE has been discussed, it is now necessary to detail how this is used to the transform the OCP in order to account for the system uncertainties.

\section{Augmented Optimal Control Problem}

In order to obtain the optimal control, $u^{*}$, for a stochastic system, the cost function must be modified in order to account for the uncertainties in the system. This is achieved by using the augmented deterministic dynamics resulting from the PCE and subsequent Galerkin projection. Consequently, the augmented system will be described by the $P+1$ states, obtained by the PCE expansion. Since we are dealing with stochastic systems, we do not minimize deterministic variables, but stochastic quantities, such as the mean, and the standard deviation. This information needs to be properly incorporated into the cost function as well. In the frame of PCE, the mean and the standard deviation are defined as:

$$
\begin{aligned}
\mu(t) & =x_{0}(t) \\
\sigma(t) & =\sqrt{\sum_{i=1}^{P} \gamma_{i} x_{i}{ }^{2}(t)}
\end{aligned}
$$

By this definition, the mean always corresponds to the first PCE coefficient, $\mathbf{x}_{0}$, whilst the standard deviation is the sum of the product of normalization factor and corresponding PCE coefficient (neglecting the mean, 
$\left.\mathbf{x}_{0}\right)$.

We can therefore define the AOCP as follows:

$$
\underbrace{A O C P}_{\text {determine } u^{*}}=\left\{\begin{aligned}
\text { minimize } \quad J & =\Phi[\mu(\mathbf{x}), \sigma(\mathbf{x})]+\int_{t_{0}}^{t_{f}} \Psi[\mu(\mathbf{x}), \sigma(\mathbf{x})] d t \\
\text { s.t. } \quad \dot{x}_{k} & =\frac{1}{<\psi_{k}^{2}(\xi)>}<\sum_{i=0}^{P} \sum_{j=0}^{P} a_{i}(t) x_{j}(t) \psi_{i}(\xi) \psi_{j}(\xi), \psi_{k}(\xi)> \\
\mathbf{x}_{0}\left(t_{0}\right) & =\left[x_{0}\left(t_{0}\right), \ldots, x_{p}\left(t_{0}\right)\right]^{T} \\
\mathbf{x}_{f}\left(t_{f}\right) & =\left[x_{0}\left(t_{f}\right), \ldots, x_{p}\left(t_{f}\right)\right]^{T}, \quad k \in[0, \ldots, P] .
\end{aligned}\right.
$$

The cost is comprised of the Mayer and Lagrange terms, as previously defined in Sec II. The specific stochastic cost formulation depends on both the original cost and the desired restrictions on mean and/or variance.

For instance, if we wish to minimize the final expectation, then this can be written in terms of the PCE in the following manner: ${ }^{10}$

$$
\begin{gathered}
\mathbb{E}\left[x^{2}\right]=\sum_{i=0}^{P} \sum_{j=0}^{P} x_{i} x_{j} \int \psi_{i}(\xi) \psi_{j}(\xi) d \xi=\mathbf{x}(t)^{T} W \mathbf{x}(t) \\
W=\operatorname{diag}\left[<\psi_{0}(\xi), \psi_{0}(\xi)>, \ldots,<\psi_{k}(\xi), \psi_{k}(\xi)>\right]
\end{gathered}
$$

where $W$ denotes the weights corresponding to the inner products (which are also given by $\gamma_{k}$ ) and $\mathbf{x}$ contains the PCE coefficients. If we now consider minimum covariance trajectories, then the variance, $\sigma^{2}(x)$ can also be written in terms of the PCE expansion as:

$$
\sigma^{2}(x)=\mathbb{E}[x-\mathbb{E}[x]]^{2}=\mathbb{E}\left[x^{2}\right]-\mathbb{E}^{2}[x]=\mathbf{x}(t)^{T} W \mathbf{x}(t)-\mathbb{E}^{2}[x]
$$

where,

$$
\begin{aligned}
\mathbb{E}[x] & =\mathbf{x}(t)^{T} F \quad \text { and } \quad F=[1,0, \ldots, 0]^{T} \\
\mathbb{E}^{2}[x] & =\mathbf{x}(t)^{T} F F^{T} \mathbf{x}(t)
\end{aligned}
$$

This can therefore be further simplified:

$$
\sigma^{2}(x)=\mathbf{x}(t)^{T}\left(W-F F^{T}\right) \mathbf{x}(t)
$$

This formulation results in the attainment of optimal control solutions for minimum expectation and/or covariance trajectories following the PCE and subsequent Galerkin projection. The relations given by Eqs. (36) and (40) can enter the cost in integral form or as a final condition.

The entire process of stochastic trajectory optimization will now be outlined, and then these principles are demonstrated using two case problems in order to convey exactly how the deterministic augmented system is obtained.

\section{Overview of Stochastic Trajectory Optimization Procedure}

The proposed framework for stochastic trajectory optimization can be summarized by the following steps which are also depicted in Fig 3:

1. Assign a PCE model to each of the uncertainties within the system.

2. Transform stochastic system of dynamical equations into the augmented deterministic equivalent.

3. Transform stochastic constraints into the augmented deterministic equivalent.

4. Quantify the stochastic cost functional (dependent of whether minimum expectation and/or covariance trajectory). 
5. Solve augmented system by NLP transcription (using SPARTAN, in this case) in order to establish the optimal control.

6. Calculate the relevant statistics for the new optimized trajectory.

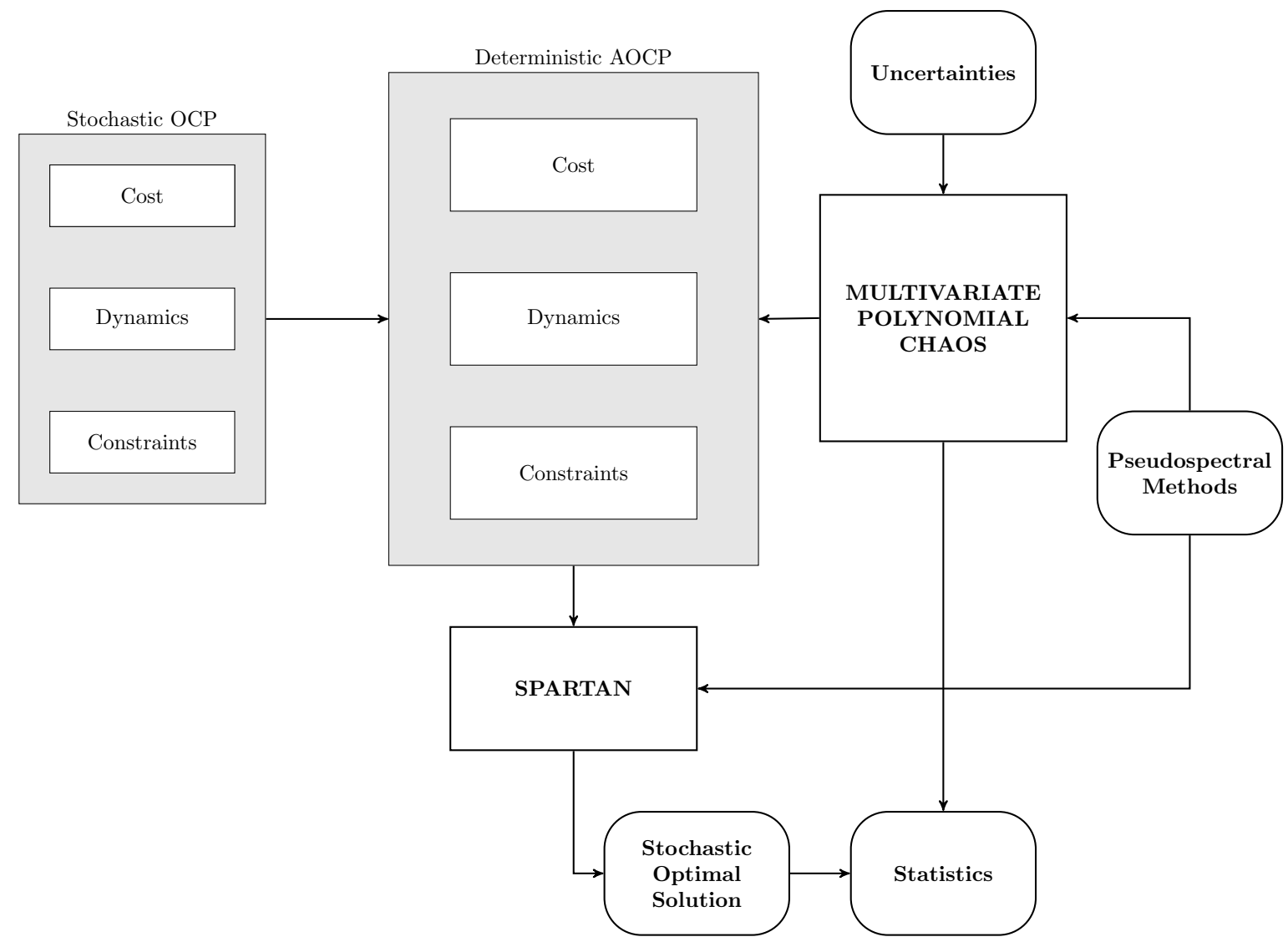

Figure 3: Proposed stochastic trajectory optimization framework based on Multivariate Polynomial Chaos and pseudospectral methods.

In the scheme depicted in Fig. 3 we can see that the stochastic OCP is transformed into an augmented OCP by means of Multivariate Polynomial Chaos. These PCE models are generated by taking into account the uncertainties within the system, and by performing the Galerkin projection required to compute the corresponding set of new differential equations. The role of pseudospectral methods is in this case twofold. First, they provide an accurate tool for the numerical integration involved in the Galerkin operation (see Appendix A and Appendix B for further details). Second, they are the core of SPARTAN, which solves the AOCP, and generates the stochastic optimal solution we are looking for. Finally, we can extract the statistics from the polynomial chaos models representing our solution by simply using Eq. (35). 


\section{Numerical Examples}

Two examples are given in order to demonstrate the capability of Multivariate Polynomial Chaos in application to stochastic trajectory optimization. First of all, the one-dimensional case is given and this is subsequently extended to multi-dimensional uncertainty. The results presented as follows were obtained for minimum final expectation and variance of the stochastic trajectory.

\section{A. Linear Example}

The first numerical example is an LTI system (as given by Eq. (32)). The problem is to minimize the norm of final state, $x\left(t_{f}\right)$, subject to time and control constraints. The optimization problem is formulated as such:

$$
\operatorname{minimize} \quad J=x^{2}\left(t_{f}\right)
$$

subject to

$$
\begin{aligned}
\dot{x}(t) & =a x(t)+b u(t) \\
\|u(t)\| & \leq 10 \\
x(0) & =2, \quad t=[0,5] \mathrm{s}
\end{aligned}
$$

where $a=1$ and $b=1$ in the deterministic case. In order to determine exactly how uncertainty impacts upon the trajectory, both parameter uncertainty and initial condition uncertainty are explored. The distribution for the parameter is normal, $a \sim N(1,0.05)$. The initial condition uncertainty has a normal distribution, $x_{0} \sim N(2,0.001)$.

First of all, one-dimensional parameter uncertainty is demonstrated in order to highlight the differences in implementation between that and the multi-dimensional problem. If $a \sim N(1,0.05)$, then for a second order expansion $(n=2)$ there will be $n+1$ coefficients, and the initial parameter PCE coefficients are $\boldsymbol{a}_{i}=[1,0.05,0]$. Considering there is no uncertainty in the initial conditions (ie., $\sigma_{x}=0$ ), the initial condition PCE coefficients are simply $\mathbf{x}_{0}=[1,0,0]^{T}$. The transformation matrix, $\mathbf{A}$, as given by Eq. (34), is determined and the augmented system of equations is then:

Remark 4 Note that PCE values have been further validated using the PCET toolbox, ${ }^{24}$ in order to verify the method and accuracy of quadrature techniques.

$$
\dot{\mathbf{x}}=\left[\begin{array}{c}
\dot{x}_{0} \\
\dot{x}_{1} \\
\dot{x}_{2}
\end{array}\right]=\left[\begin{array}{ccc}
1 & 0.05 & 0 \\
0.05 & 1 & 0.1 \\
0 & 0.05 & 1
\end{array}\right]\left[\begin{array}{l}
x_{0} \\
x_{1} \\
x_{2}
\end{array}\right]+\left[\begin{array}{l}
1 \\
0 \\
0
\end{array}\right] u
$$

As detailed in the previous section, we can define the cost function in terms of the PCE by using the coefficients, $\mathbf{x}$ :

$$
J_{A O C P}=\underbrace{\gamma_{0} x_{0}^{2}+\gamma_{1} x_{1}^{2}+\gamma_{2} x_{2}^{2}}_{\mathbb{E}\left[x^{2}\right]}+\underbrace{\gamma_{1} x_{1}^{2}+\gamma_{2} x_{2}^{2}}_{\mathbb{E}^{2}[x]} \quad \gamma_{k}=[1,1,2]^{T}
$$

This AOCP is then solved by SPARTAN, resulting in a stochastic optimal trajectory. The AOCP now involves minimizing the square of expectation and standard deviation (i.e., variance). Now, in order to establish whether the new control profile is in fact more robust in the presence of this uncertainty, a Monte Carlo (MC) analysis is performed. Both the original deterministic control solution and the new stochastic control solutions are used to propagate the perturbed system represented by Eq. (42). The mean $\mu$, and standard deviation, $\sigma$, are calculated for both cases. The control profiles can be seen in Fig. 4(a), whilst the corresponding PCE coefficients are shown in Fig. 4(b). Finally, the MC analysis consisting of 1000 runs is depicted in Fig. 5, where it is very apparent that the newly obtained stochastic control generates trajectories which are far less perturbed than in the deterministic case. Considering that this linear system is inherently unstable, it is rather impressive that the expectation converges to a minimum value of 0.07528 using the new stochastic control, in comparison to 2.4589 in the deterministic case. It is apparent that the original control solution offers no robustness to the uncertainty; exhibiting wide divergence (with a standard deviation equal to 11.8551 ).

Now if both parameter and initial condition uncertainty are considered, the initial parameter PCE coefficients are $\boldsymbol{a}_{i}=[1,0,0.05,0,0,0]$ and $\mathbf{x}_{0}=[2,0.001,0,0,0,0]^{T}$. The augmented system to be solved is defined 


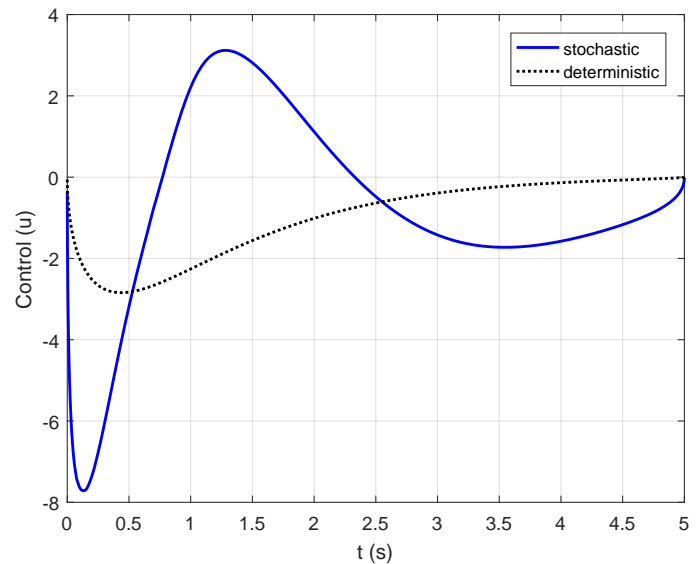

(a) Control for 1-D linear problem.

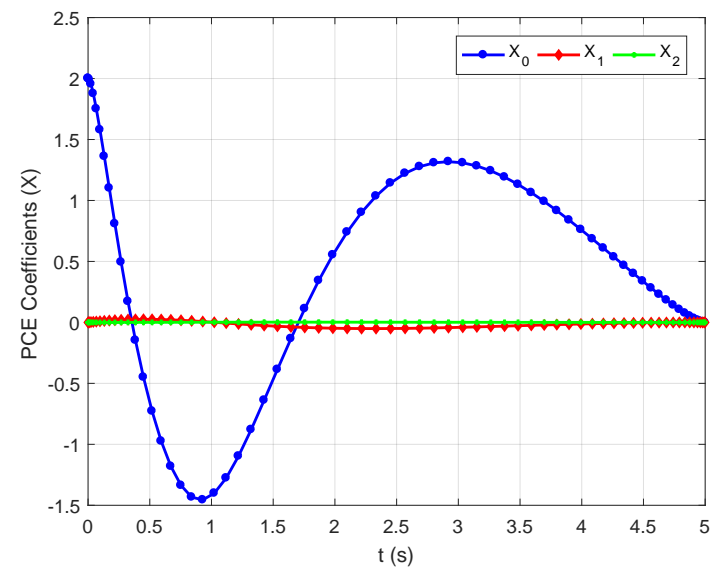

(b) PCE coefficients for 1-D linear problem.

Figure 4: Control and PCE Coefficients for 1-D linear problem (a) Control, (b) PCE coefficients.

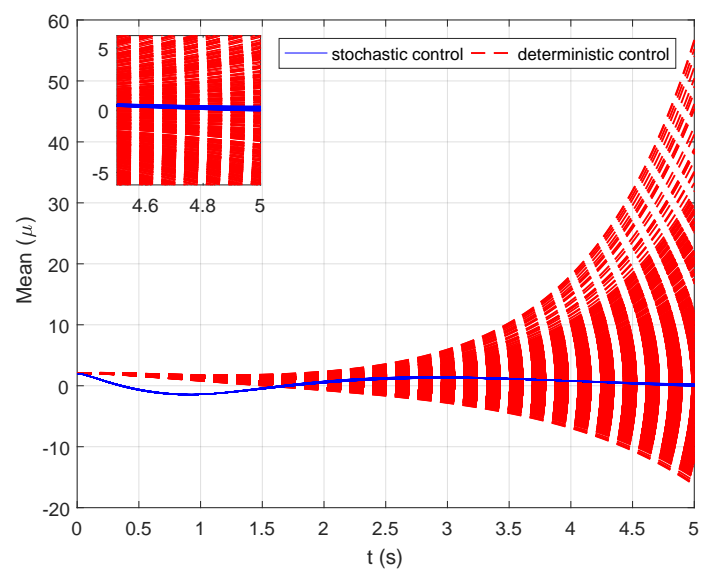

Figure 5: MC analysis for 1-D linear problem.

as follows, noting that the AOCP will have the same form as before, but will include the additional PCE coefficients and normalization factors, $\gamma_{k}$.

$$
\dot{\mathbf{x}}=\left[\begin{array}{c}
\dot{x}_{0} \\
\dot{x}_{1} \\
\dot{x}_{2} \\
\dot{x}_{3} \\
\dot{x}_{4} \\
\dot{x}_{5}
\end{array}\right]=\left[\begin{array}{cccccc}
1 & 0 & 0.05 & 0 & 0 & 0 \\
0 & 1 & 0 & 0 & 0.05 & 0 \\
0.05 & 0 & 1 & 0 & 0 & 0.1 \\
0 & 0 & 0 & 1 & 0 & 0 \\
0 & 0.05 & 0 & 0 & 1 & 0 \\
0 & 0 & 0.05 & 0 & 0 & 1
\end{array}\right]\left[\begin{array}{l}
x_{0} \\
x_{1} \\
x_{2} \\
x_{3} \\
x_{4} \\
x_{5}
\end{array}\right]+\left[\begin{array}{l}
1 \\
0 \\
0 \\
0 \\
0 \\
0
\end{array}\right] u
$$

For the two-dimensional problem $\gamma_{k}=[1,1,1,2,1,2]^{T}$. The resultant control is shown in comparison to the one-dimensional and deterministic cases (Fig. 6(a)), as are the coefficients (Fig. 6(b)). The MC analysis is given in Fig. 7 and demonstrates that uncertainty in the initial condition results in a slightly degraded response due to the dispersion at the end. Although there is a comparatively higher std of $1.5578 \cdot 10^{0}$ in the two-dimensional case, it is still a significant reduction when compared to the deterministic std of $1.1947 \cdot 10^{1}$. The mean also increases for the multi-dimensional case - now it is $1.6030 \cdot 10^{-1}$ using the stochastic control. Despite this, it is still very apparent that the augmented optimal control solution is much less sensitive to the uncertainties than the deterministic control, as the final expectation and standard deviation are between 
one and three orders of magnitude smaller than that of the original solution.

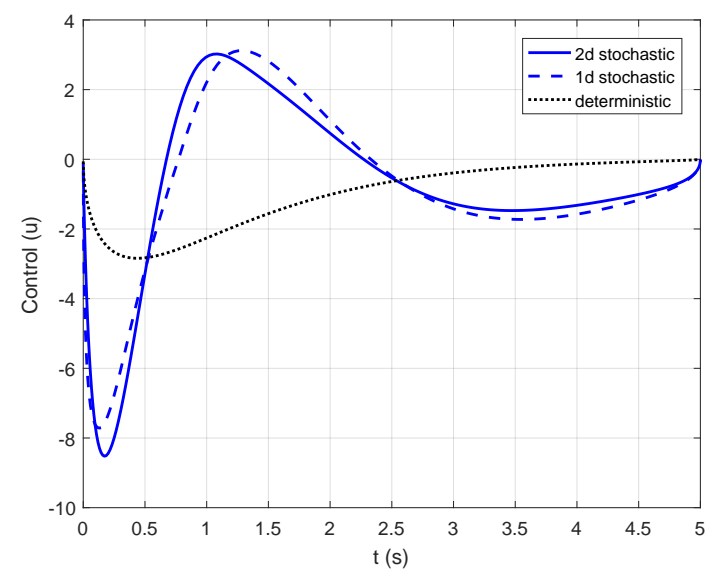

(a) Control for 2-D linear problem.

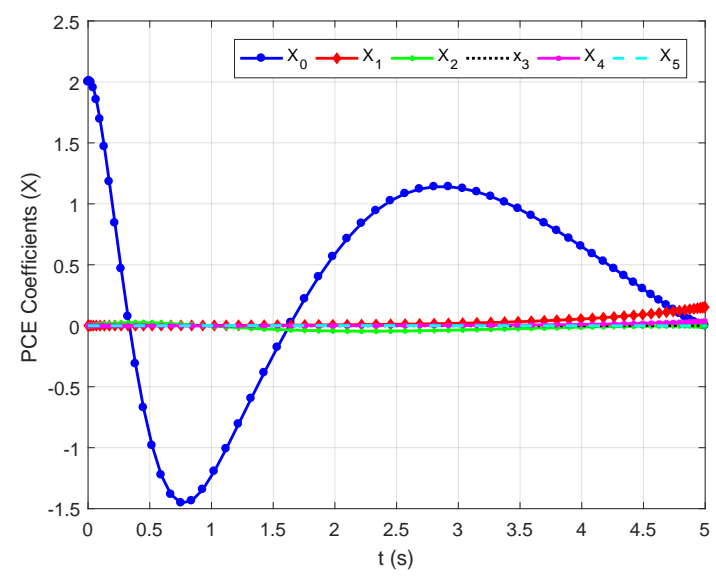

(b) PCE coefficients for 2-D linear problem.

Figure 6: Control and PCE Coefficients for 2-D linear problem (a) Control, (b) PCE coefficients

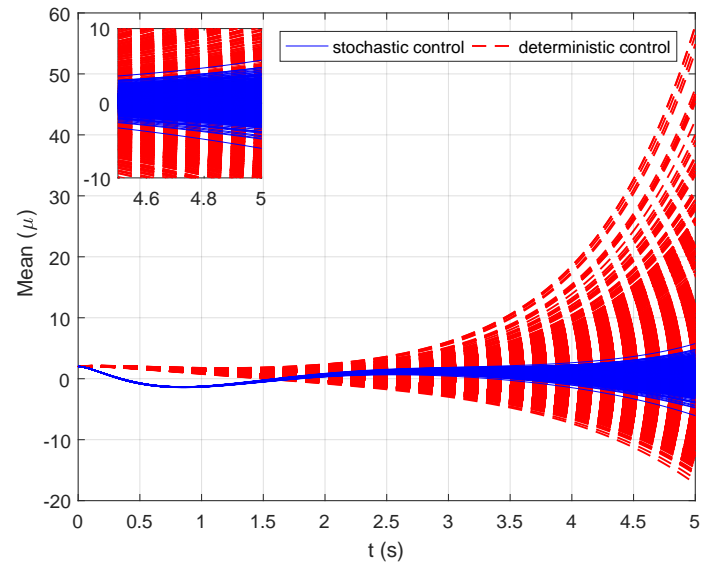

Figure 7: MC analysis for 2-D linear problem.

A comparison between the solutions obtained for the one and two-dimensional linear problems is presented in Tab. 3. From this it becomes evident that the optimized stochastic control solution shows a significant reduction in final expectation and variance when compared to the original control; thus, emphasizing the benefit of the stochastic AOCP.

Table 3: Comparison of results for 1-D and 2-D linear problems.

\begin{tabular}{c|cc|cc}
\hline \hline \multirow{2}{*}{ Case } & \multicolumn{2}{|c|}{ 1-D } & \multicolumn{2}{c}{2 -D } \\
\cline { 2 - 5 } & Mean $\mu$ & Std $\sigma$ & Mean $\mu$ & Std $\sigma$ \\
\hline Deterministic & $2.4689 \cdot 10^{0}$ & $1.1855 \cdot 10^{1}$ & $2.5556 \cdot 10^{0}$ & $1.1947 \cdot 10^{1}$ \\
\hline Stochastic & $7.5280 \cdot 10^{-2}$ & $3.0755 \cdot 10^{-2}$ & $1.6030 \cdot 10^{-1}$ & $1.5578 \cdot 10^{0}$ \\
\hline \hline
\end{tabular}




\section{B. Non-linear Example}

The next example is a non-linear case,${ }^{19}$ where the cost function is represented by a Mayer term:

$$
\text { minimize } \quad J=-x\left(t_{f}\right)
$$

subject to

$$
\begin{aligned}
& \dot{x}(t)=a\left(-x(t)+x(t) u(t)-u(t)^{2}\right) \\
& x(0)=1, \quad t_{f}=[0,2] \mathrm{s}
\end{aligned}
$$

The stochastic quantities are $a \sim N(2.5,0.1)$ and $x_{0} \sim U(0.9,1.1)$ i.e., normal parameter and uniform initial condition with lower bound, $l_{b}$, and upper bound, $u_{b}$. For the uniform distribution, $\mu=\frac{1}{2}\left(l_{b}+u_{b}\right)$ and $\sigma=\frac{1}{2}\left(u_{b}-l_{b}\right)$. This example differs from the previous, not only in the non-linearity aspect, but also in the fact that the control is affected by the stochastic parameter. The initial parameter coefficients are $\boldsymbol{a}_{i}=[2.5,0.1,0]$ and the initial conditions are $\mathbf{x}_{0}=[1,0,0]^{T}$. The augmented stochastic system is therefore:

$$
\dot{\mathbf{x}}(t)=-\mathbf{A} \mathbf{x}(t)+u(t) \mathbf{A} \mathbf{x}(t)-\mathbf{B} u(t)^{2}
$$

In the case of one-dimensional parameter uncertainty,

$$
\mathbf{A}=\left[\begin{array}{ccc}
2.5 & 0.1 & 0 \\
0.1 & 2.5 & 0.2 \\
0 & 0.1 & 2.5
\end{array}\right] \quad \text { and } \quad \mathbf{B}=\left[\begin{array}{c}
2.5 \\
0.1 \\
0
\end{array}\right]
$$

The AOCP to be solved for a minimum expectation and variance trajectory is as such:

$$
J_{A O C P}=\underbrace{\gamma_{0} x_{0}+\gamma_{1} x_{1}+\gamma_{2} x_{2}}_{\mathbb{E}\left[x^{2}\right]}+\underbrace{\sqrt{\gamma_{1} x_{1}^{2}+\gamma_{2} x_{2}^{2}}}_{\mathbb{E}^{2}[x]} \quad \gamma_{k}=[1,1,2]^{T}
$$

A comparison of the obtained optimal solution and the original is shown in Fig. 8(a) and the corresponding PCE coefficients, $\mathbf{x}$, are shown in Fig. 8(b). As before, a MC analysis of 1000 runs is performed in order to establish whether the newly optimized control is more robust in regard to the uncertainty (Fig. 9). The stochastic solution converges to a final expectation of $-4.0212 \cdot 10^{-3}$ - more than half that of the deterministic case. The solution also exhibits a much higher final convergence (with a standard deviation of $9.4488 \cdot 10^{-5}$, compared to $\left.1.8436 \cdot 10^{-3}\right)$. These results are shown in Tab. 4 .

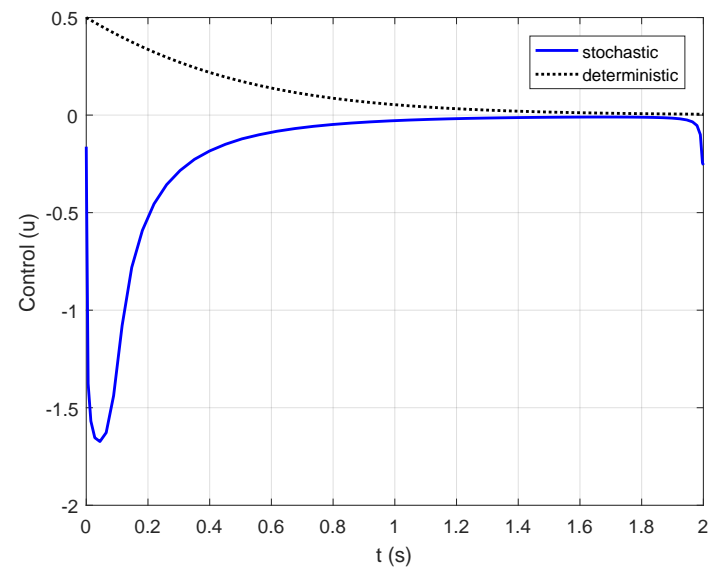

(a) Control for 1-D non-linear problem.

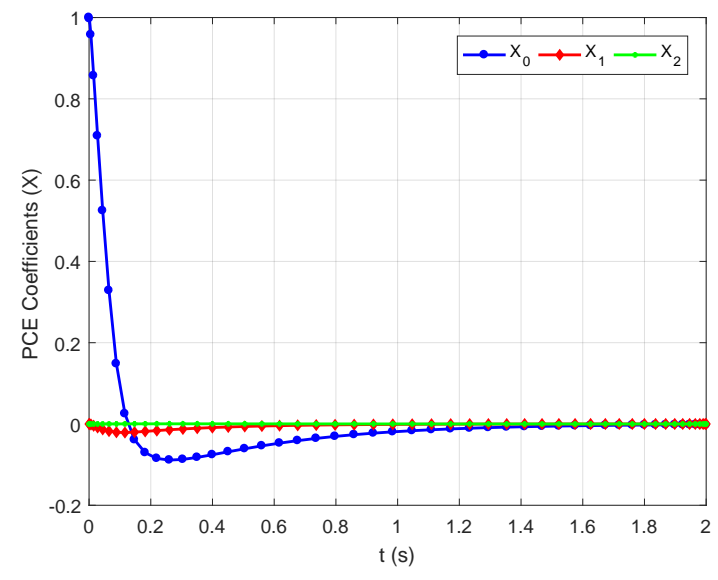

(b) PCE Coefficients for 1-D non-linear problem.

Figure 8: Control and PCE Coefficients for 1-D non-linear problem (a) Control, (b) PCE coefficients.

For the two-dimensional case, the transformation matrices are given by Eq. (51). The initial PCE coefficients for parameter and initial condition are $\boldsymbol{a}_{i}=[2.5,0,0.1,0,0,0]$ and $\mathbf{x}_{0}=[1,0.1,0,0,0,0]^{T}$, respectively. The 


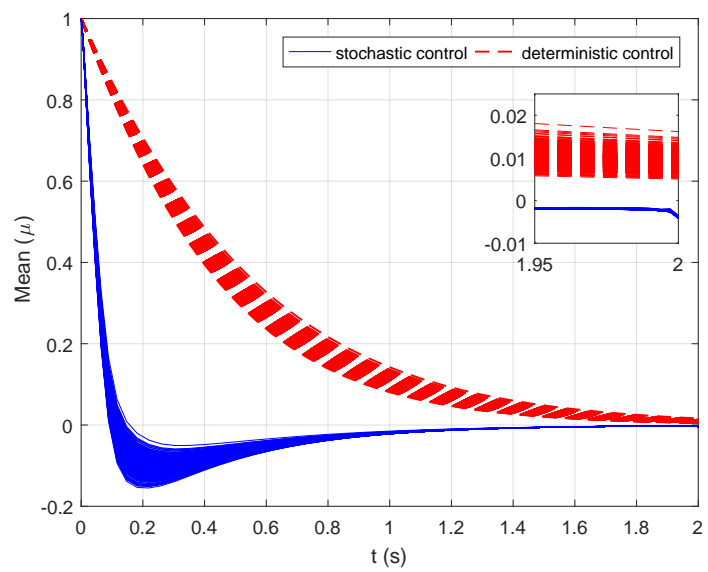

Figure 9: MC analysis for 1-D non-linear problem.

AOCP will be as in Eq. (50), but with the additional coefficients. Note that the normalization factors are now $\gamma_{k}=\left[1, \frac{1}{3}, 1, \frac{1}{5}, \frac{1}{3}, 3\right]^{T}$.

$$
\mathbf{A}=\left[\begin{array}{cccccc}
2.5 & 0 & 0.1 & 0 & 0 & 0 \\
0 & 2.5 & 0 & 0 & 0.1 & 0 \\
0.1 & 0 & 2.5 & 0 & 0 & 0.2 \\
0 & 0 & 0 & 2.5 & 0 & 0 \\
0 & 0.1 & 0 & 0 & 2.5 & 0 \\
0 & 0 & 0.1 & 0 & 0 & 2.5
\end{array}\right] \text { and } \mathbf{B}=\left[\begin{array}{c}
2.5 \\
0 \\
0.1 \\
0 \\
0 \\
0
\end{array}\right]
$$

The control profile resulting from uncertainties in both the parameter and the initial conditions is shown in Fig. 10(a), along with the one-dimensional and deterministic cases. The corresponding PCE coefficients are given in Fig. 10(b). Again it is noted that there are six coefficients due to the fact that it is a second order expansion, with two stochastic variables. The uncertainty in initial condition is very obvious in both the stochastic and deterministic case, however, the former converges very nicely to a final expectation of $-4.4482 \cdot 10^{-4}$. Although this result seems to be better than the 1-D case, this is not completely true. In fact, the standard deviation worsens by one order of magnitude to $3.4580 \cdot 10^{-4}$. Nevertheless, the method still outperforms the deterministic approach by two orders of magnitude.

A full comparison of the statistics for the one-dimensional and two-dimensional non-linear problems is shown in Tab. 4.

Table 4: Comparison of results for 1-D and 2-D non-linear problems.

\begin{tabular}{c|cc|cc}
\hline \hline \multirow{2}{*}{ Case } & \multicolumn{2}{|c|}{$1-\mathrm{D}$} & \multicolumn{2}{c}{$2-\mathrm{D}$} \\
\cline { 2 - 5 } & Mean $\mu$ & Std $\sigma$ & Mean $\mu$ & Std $\sigma$ \\
\hline Deterministic & $9.1312 \cdot 10^{-3}$ & $1.8436 \cdot 10^{-3}$ & $9.2107 \cdot 10^{-3}$ & $2.2081 \cdot 10^{-3}$ \\
\hline Stochastic & $-4.0212 \cdot 10^{-3}$ & $9.4488 \cdot 10^{-5}$ & $-4.4482 \cdot 10^{-4}$ & $3.4580 \cdot 10^{-4}$ \\
\hline \hline
\end{tabular}




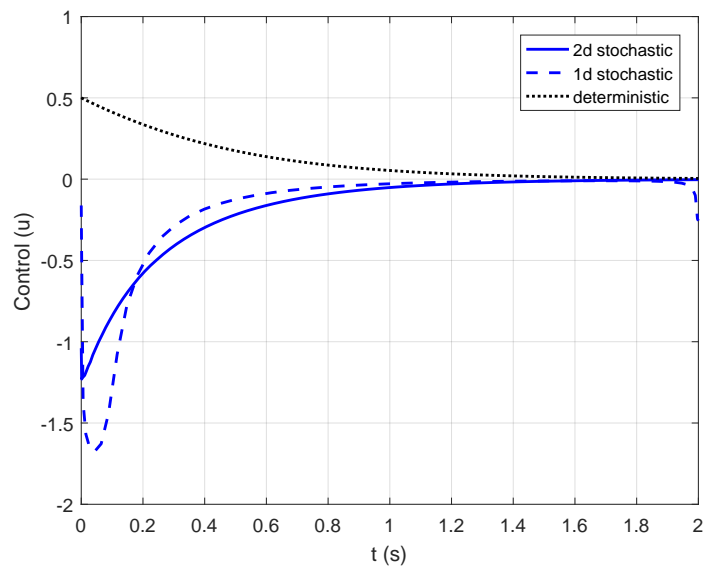

(a) Control for 2-D non-linear problem.

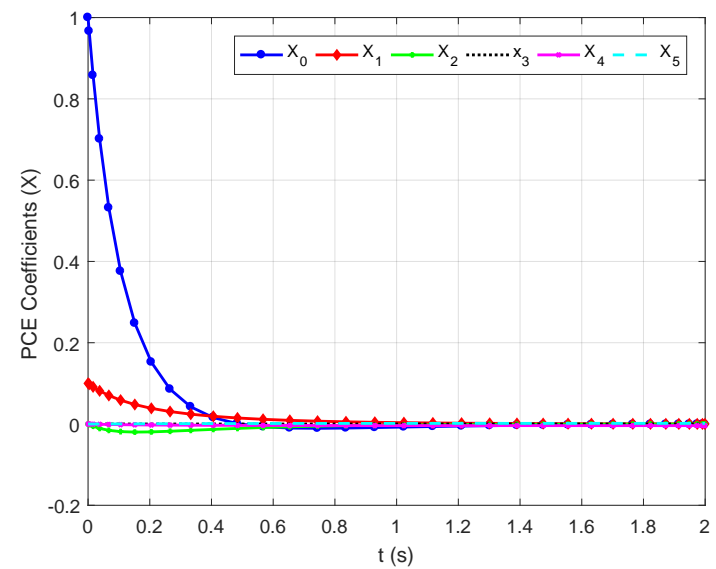

(b) PCE Coefficients for 2-D non-linear problem.

Figure 10: Control and PCE Coefficients for 2-D non-linear problem (a) Control (b) PCE coefficients.

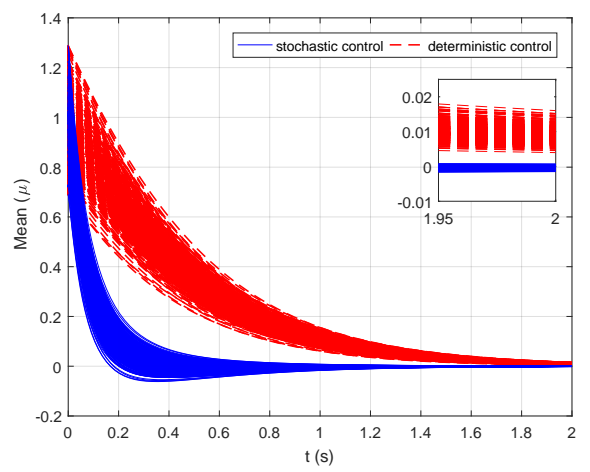

Figure 11: MC analysis for 2-D non-linear problem.

\section{Conclusions}

It has been demonstrated how to obtain optimal trajectories in the presence of uncertainties. Multivariate polynomial chaos is a very effective method for uncertainty propagation, offering accurate results, without too heavy computational implications. Uncertainty propagation was performed using PCE in order to obtain the augmented deterministic system. The original cost functions were transformed into corresponding ones which minimize stochastic quantities, such as the mean and/or standard deviation. This system was subsequently solved using NLP transcription, performed by SPARTAN, in order to obtain the stochastic optimal trajectory. These principles were demonstrated using two examples, with both the one-dimensional and multi-dimensional cases analyzed. This has been done in order to convey the benefits of gPC in application to optimal trajectory generation problems.

Results demonstrate an improvement in performance with respect to the deterministic classic approach. This framework in fact allows us to control the mean and standard deviation significantly under uncertainties; the examples presented show improvements by several orders of magnitude when the optimized stochastic control solution is utilized. The proposed framework leads to open-loop trajectories far more robust to uncertainties in the initial conditions and/or parameters. Future work will involve application to higherdimension problems and also incorporate stochastic path constraints. Moreover, the impact of the strategy proposed here on feedback control laws will be explored. 


\section{Appendix A - 1-D Polynomial Chaos Expansion for Linear Systems}

The derivation presented here represents a one-dimensional uncertainty for linear systems (as given by Eq. (32)). Due to the univariate nature of the problem, the order of expansion is simply related to the highest order of polynomial, i.e. $n+1$. If we consider a second order expansion, there there will be three resulting $\mathrm{PCE}$ coefficients in the augmented system and hence, $\mathbf{A}$ is a $[3 \times 3]$ matrix. The subscript of each univariate polynomial base, $\psi$, is the single index, $i, j, k$, respectively and conveys the order of the polynomial.

$$
\dot{\mathbf{x}}=\left[\begin{array}{l}
\dot{x}_{0} \\
\dot{x}_{1} \\
\dot{x}_{2}
\end{array}\right]=\left[\begin{array}{lll}
A_{00} & A_{01} & A_{02} \\
A_{10} & A_{11} & A_{12} \\
A_{20} & A_{21} & A_{22}
\end{array}\right]\left[\begin{array}{l}
x_{0} \\
x_{1} \\
x_{2}
\end{array}\right]
$$

where,

$\dot{x}_{0} \quad$ terms $\left\{\begin{array}{l}\gamma_{0}=<\psi_{0}^{2}(\xi)>=\int \psi_{0}(\xi) \psi_{0}(\xi) \\ A_{00}=\frac{1}{\gamma_{0}}\left[a_{0} \int \psi_{0}(\xi) \psi_{0}(\xi) \psi_{0}(\xi)+a_{1} \int \psi_{1}(\xi) \psi_{0}(\xi) \psi_{0}(\xi)+a_{2} \int \psi_{2}(\xi) \psi_{0}(\xi) \psi_{0}(\xi)\right] \\ A_{01}=\frac{1}{\gamma_{0}}\left[a_{0} \int \psi_{0}(\xi) \psi_{1}(\xi) \psi_{0}(\xi)+a_{1} \int \psi_{1}(\xi) \psi_{1}(\xi) \psi_{0}(\xi)+a_{2} \int \psi_{2}(\xi) \psi_{1}(\xi) \psi_{0}(\xi)\right] \\ A_{02}=\frac{1}{\gamma_{0}}\left[a_{0} \int \psi_{0}(\xi) \psi_{2}(\xi) \psi_{0}(\xi)+a_{1} \int \psi_{1}(\xi) \psi_{2}(\xi) \psi_{0}(\xi)+a_{2} \int \psi_{2}(\xi) \psi_{2}(\xi) \psi_{0}(\xi)\right]\end{array}\right.$

$\dot{x}_{1} \quad$ terms $\left\{\begin{array}{l}\gamma_{1}=<\psi_{1}^{2}(\xi)>=\int \psi_{1}(\xi) \psi_{1}(\xi) \\ A_{10}=\frac{1}{\gamma_{1}}\left[a_{0} \int \psi_{0}(\xi) \psi_{0}(\xi) \psi_{1}(\xi)+a_{1} \int \psi_{1}(\xi) \psi_{0}(\xi) \psi_{1}(\xi)+a_{2} \int \psi_{2}(\xi) \psi_{0}(\xi) \psi_{1}(\xi)\right] \\ A_{11}=\frac{1}{\gamma_{1}}\left[a_{0} \int \psi_{0}(\xi) \psi_{1}(\xi) \psi_{1}(\xi)+a_{1} \int \psi_{1}(\xi) \psi_{1}(\xi) \psi_{1}(\xi)+a_{2} \int \psi_{2}(\xi) \psi_{1}(\xi) \psi_{1}(\xi)\right] \\ A_{12}=\frac{1}{\gamma_{1}}\left[a_{0} \int \psi_{0}(\xi) \psi_{2}(\xi) \psi_{1}(\xi)+a_{1} \int \psi_{1}(\xi) \psi_{2}(\xi) \psi_{1}(\xi)+a_{2} \int \psi_{2}(\xi) \psi_{1}(\xi) \psi_{1}(\xi)\right]\end{array}\right.$

$\dot{x}_{2}$ terms $\left\{\begin{array}{l}\gamma_{2}=<\psi_{2}^{2}(\xi)>=\int \psi_{2}(\xi) \psi_{2}(\xi) \\ A_{20}=\frac{1}{\gamma_{2}}\left[a_{0} \int \psi_{0}(\xi) \psi_{0}(\xi) \psi_{2}(\xi)+a_{1} \int \psi_{1}(\xi) \psi_{0}(\xi) \psi_{2}(\xi)+a_{2} \int \psi_{2}(\xi) \psi_{0}(\xi) \psi_{2}(\xi)\right] \\ A_{21}=\frac{1}{\gamma_{2}}\left[a_{0} \int \psi_{0}(\xi) \psi_{1}(\xi) \psi_{2}(\xi)+a_{1} \int \psi_{1}(\xi) \psi_{0}(\xi) \psi_{2}(\xi)+a_{2} \int \psi_{2}(\xi) \psi_{0}(\xi) \psi_{2}(\xi)\right] \\ A_{22}=\frac{1}{\gamma_{2}}\left[a_{0} \int \psi_{0}(\xi) \psi_{2}(\xi) \psi_{2}(\xi)+a_{1} \int \psi_{1}(\xi) \psi_{2}(\xi) \psi_{2}(\xi)+a_{2} \int \psi_{2}(\xi) \psi_{2}(\xi) \psi_{2}(\xi)\right]\end{array}\right.$

Note that the third component in each $\mathbf{A}$ term is zero since the PCE coefficient matrix is $\boldsymbol{a}_{i}=\left[\mu_{a}, \sigma_{a}, 0\right]$ for one-dimensional parameter uncertainty. It also follows that these relationships are further simplified for the case of initial condition uncertainty due to the fact that the only non-zero initial PCE coefficient is $\mu_{a}$.

\section{Appendix B - 2-D Polynomial Chaos Expansion for Linear Systems}

In order to fully demonstrate how the Multivariate PC system is derived, the full integral solution for a two-dimensional problem is given. This is for an order of expansion, $n=2$, and since there are two random variables i.e. $d=2$, there are six PCE coefficients $(P=6)$. The subscript of each polynomial base corresponds to the multi-index and the notation has been shortened for the multivariate polynomial basis so that for e.g., $\psi_{10}(\xi)=\psi_{1}\left(\xi_{1}\right) \psi_{0}\left(\xi_{2}\right)$. Where $\psi_{1}\left(\xi_{1}\right)$ is the $1^{\text {st }}$ order univariate polynomial corresponding to the distribution of the parameter, whilst $\psi_{0}\left(\xi_{2}\right)$ is a univariate polynomial of order zero related to the distribution of the initial condition.

$$
\dot{\mathbf{x}}=\left[\begin{array}{c}
\dot{x}_{0} \\
\dot{x}_{1} \\
\dot{x}_{2} \\
\dot{x}_{3} \\
\dot{x}_{4} \\
\dot{x}_{5}
\end{array}\right]=\left[\begin{array}{llllll}
A_{00} & A_{01} & A_{02} & A_{03} & A_{04} & A_{05} \\
A_{10} & A_{11} & A_{12} & A_{13} & A_{14} & A_{15} \\
A_{20} & A_{21} & A_{22} & A_{23} & A_{24} & A_{25} \\
A_{30} & A_{31} & A_{32} & A_{33} & A_{34} & A_{35} \\
A_{40} & A_{41} & A_{42} & A_{43} & A_{44} & A_{45} \\
A_{50} & A_{51} & A_{52} & A_{53} & A_{54} & A_{55}
\end{array}\right]\left[\begin{array}{c}
x_{0} \\
x_{1} \\
x_{2} \\
x_{3} \\
x_{4} \\
x_{5}
\end{array}\right]
$$


where,

$\dot{x}_{0} \quad$ terms $\left\{\begin{array}{l}\gamma_{0}=<\psi_{00}^{2}(\xi)>=\int \psi_{00}(\xi) \psi_{00}(\xi) \\ A_{00}=\frac{1}{\gamma_{0}}\left[a_{0} \int \psi_{00}(\xi) \psi_{00}(\xi) \psi_{00}(\xi)+a_{2} \int \psi_{01}(\xi) \psi_{00}(\xi) \psi_{00}(\xi)\right] \\ A_{01}=\frac{1}{\gamma_{0}}\left[a_{0} \int \psi_{00}(\xi) \psi_{10}(\xi) \psi_{00}(\xi)+a_{2} \int \psi_{01}(\xi) \psi_{10}(\xi) \psi_{00}(\xi)\right] \\ A_{02}=\frac{1}{\gamma_{0}}\left[a_{0} \int \psi_{00}(\xi) \psi_{01}(\xi) \psi_{00}(\xi)+a_{2} \int \psi_{01}(\xi) \psi_{01}(\xi) \psi_{00}(\xi)\right] \\ A_{03}=\frac{1}{\gamma_{0}}\left[a_{0} \int \psi_{00}(\xi) \psi_{20}(\xi) \psi_{00}(\xi)+a_{2} \int \psi_{01}(\xi) \psi_{20}(\xi) \psi_{00}(\xi)\right] \\ A_{04}=\frac{1}{\gamma_{0}}\left[a_{0} \int \psi_{00}(\xi) \psi_{11}(\xi) \psi_{00}(\xi)+a_{2} \int \psi_{01}(\xi) \psi_{11}(\xi) \psi_{00}(\xi)\right] \\ A_{05}=\frac{1}{\gamma_{0}}\left[a_{0} \int \psi_{00}(\xi) \psi_{02}(\xi) \psi_{00}(\xi)+a_{2} \int \psi_{01}(\xi) \psi_{02}(\xi) \psi_{00}(\xi)\right]\end{array}\right.$

$\dot{x}_{1}$ terms $\left\{\begin{array}{l}\gamma_{1}=<\psi_{10}^{2}(\xi)>=\int \psi_{10}(\xi) \psi_{10}(\xi) \\ A_{10}=\frac{1}{\gamma_{1}}\left[a_{0} \int \psi_{00}(\xi) \psi_{00}(\xi) \psi_{10}(\xi)+a_{2} \int \psi_{01}(\xi) \psi_{00}(\xi) \psi_{10}(\xi)\right] \\ A_{11}=\frac{1}{\gamma_{1}}\left[a_{0} \int \psi_{00}(\xi) \psi_{10}(\xi) \psi_{10}(\xi)+a_{2} \int \psi_{01}(\xi) \psi_{10}(\xi) \psi_{10}(\xi)\right] \\ A_{12}=\frac{1}{\gamma_{1}}\left[a_{0} \int \psi_{00}(\xi) \psi_{01}(\xi) \psi_{10}(\xi)+a_{2} \int \psi_{01}(\xi) \psi_{01}(\xi) \psi_{10}(\xi)\right] \\ A_{13}=\frac{1}{\gamma_{1}}\left[a_{0} \int \psi_{00}(\xi) \psi_{20}(\xi) \psi_{10}(\xi)+a_{2} \int \psi_{01}(\xi) \psi_{20}(\xi) \psi_{10}(\xi)\right] \\ A_{14}=\frac{1}{\gamma_{1}}\left[a_{0} \int \psi_{00}(\xi) \psi_{11}(\xi) \psi_{10}(\xi)+a_{2} \int \psi_{01}(\xi) \psi_{11}(\xi) \psi_{10}(\xi)\right] \\ A_{15}=\frac{1}{\gamma_{1}}\left[a_{0} \int \psi_{00}(\xi) \psi_{02}(\xi) \psi_{10}(\xi)+a_{2} \int \psi_{01}(\xi) \psi_{02}(\xi) \psi_{10}(\xi)\right]\end{array}\right.$

$\dot{x}_{2}$ terms $\begin{cases}\gamma_{2} & =<\psi_{01}^{2}(\xi)>=\int \psi_{01}(\xi) \psi_{01}(\xi) \\ A_{20} & =\frac{1}{\gamma_{2}}\left[a_{0} \int \psi_{00}(\xi) \psi_{00}(\xi) \psi_{01}(\xi)+a_{2} \int \psi_{01}(\xi) \psi_{00}(\xi) \psi_{01}(\xi)\right] \\ A_{21} & =\frac{1}{\gamma_{2}}\left[a_{0} \int \psi_{00}(\xi) \psi_{10}(\xi) \psi_{01}(\xi)+a_{2} \int \psi_{01}(\xi) \psi_{10}(\xi) \psi_{01}(\xi)\right] \\ A_{22} & =\frac{1}{\gamma_{2}}\left[a_{0} \int \psi_{00}(\xi) \psi_{01}(\xi) \psi_{01}(\xi)+a_{2} \int \psi_{01}(\xi) \psi_{01}(\xi) \psi_{01}(\xi)\right] \\ A_{23} & =\frac{1}{\gamma_{2}}\left[a_{0} \int \psi_{00}(\xi) \psi_{20}(\xi) \psi_{01}(\xi)+a_{2} \int \psi_{01}(\xi) \psi_{20}(\xi) \psi_{01}(\xi)\right] \\ A_{24} & =\frac{1}{\gamma_{2}}\left[a_{0} \int \psi_{00}(\xi) \psi_{11}(\xi) \psi_{01}(\xi)+a_{2} \int \psi_{01}(\xi) \psi_{11}(\xi) \psi_{01}(\xi)\right] \\ A_{25} & =\frac{1}{\gamma_{2}}\left[a_{0} \int \psi_{00}(\xi) \psi_{02}(\xi) \psi_{01}(\xi)+a_{2} \int \psi_{01}(\xi) \psi_{02}(\xi) \psi_{01}(\xi)\right]\end{cases}$

$\dot{x}_{3}$ terms $\left\{\begin{aligned} \gamma_{3} & =<\psi_{20}^{2}(\xi)>=\int \psi_{20}(\xi) \psi_{20}(\xi) \\ A_{30} & =\frac{1}{\gamma_{3}}\left[a_{0} \int \psi_{00}(\xi) \psi_{00}(\xi) \psi_{20}(\xi)+a_{2} \int \psi_{01}(\xi) \psi_{00}(\xi) \psi_{20}(\xi)\right] \\ A_{31} & =\frac{1}{\gamma_{3}}\left[a_{0} \int \psi_{00}(\xi) \psi_{10}(\xi) \psi_{20}(\xi)+a_{2} \int \psi_{01}(\xi) \psi_{10}(\xi) \psi_{20}(\xi)\right] \\ A_{32} & =\frac{1}{\gamma_{3}}\left[a_{0} \int \psi_{00}(\xi) \psi_{01}(\xi) \psi_{20}(\xi)+a_{2} \int \psi_{01}(\xi) \psi_{01}(\xi) \psi_{20}(\xi)\right] \\ A_{33} & =\frac{1}{\gamma_{3}}\left[a_{0} \int \psi_{00}(\xi) \psi_{20}(\xi) \psi_{20}(\xi)+a_{2} \int \psi_{01}(\xi) \psi_{20}(\xi) \psi_{20}(\xi)\right] \\ A_{34} & =\frac{1}{\gamma_{3}}\left[a_{0} \int \psi_{00}(\xi) \psi_{11}(\xi) \psi_{20}(\xi)+a_{2} \int \psi_{01}(\xi) \psi_{11}(\xi) \psi_{20}(\xi)\right] \\ A_{35} & =\frac{1}{\gamma_{3}}\left[a_{0} \int \psi_{00}(\xi) \psi_{02}(\xi) \psi_{20}(\xi)+a_{2} \int \psi_{01}(\xi) \psi_{02}(\xi) \psi_{20}(\xi)\right]\end{aligned}\right.$

$\dot{x}_{4}$ terms $\begin{cases}\gamma_{4} & =<\psi_{11}^{2}(\xi)>=\int \psi_{11}(\xi) \psi_{11}(\xi) \\ A_{40} & =\frac{1}{\gamma_{4}}\left[a_{0} \int \psi_{00}(\xi) \psi_{00}(\xi) \psi_{11}(\xi)+a_{2} \int \psi_{01}(\xi) \psi_{00}(\xi) \psi_{11}(\xi)\right] \\ A_{41} & =\frac{1}{\gamma_{4}}\left[a_{0} \int \psi_{00}(\xi) \psi_{10}(\xi) \psi_{11}(\xi)+a_{2} \int \psi_{01}(\xi) \psi_{10}(\xi) \psi_{11}(\xi)\right] \\ A_{42} & =\frac{1}{\gamma_{4}}\left[a_{0} \int \psi_{00}(\xi) \psi_{01}(\xi) \psi_{11}(\xi)+a_{2} \int \psi_{01}(\xi) \psi_{01}(\xi) \psi_{11}(\xi)\right] \\ A_{43} & =\frac{1}{\gamma_{4}}\left[a_{0} \int \psi_{00}(\xi) \psi_{20}(\xi) \psi_{11}(\xi)+a_{2} \int \psi_{01}(\xi) \psi_{20}(\xi) \psi_{11}(\xi)\right] \\ A_{44} & =\frac{1}{\gamma_{4}}\left[a_{0} \int \psi_{00}(\xi) \psi_{11}(\xi) \psi_{11}(\xi)+a_{2} \int \psi_{01}(\xi) \psi_{11}(\xi) \psi_{11}(\xi)\right] \\ A_{45} & =\frac{1}{\gamma_{4}}\left[a_{0} \int \psi_{00}(\xi) \psi_{02}(\xi) \psi_{11}(\xi)+a_{2} \int \psi_{01}(\xi) \psi_{02}(\xi) \psi_{11}(\xi)\right]\end{cases}$ 


$$
\dot{x}_{5} \text { terms }\left\{\begin{array}{l}
\gamma_{5}=<\psi_{02}^{2}(\xi)>=\int \psi_{02}(\xi) \psi_{02}(\xi) \\
A_{50}=\frac{1}{\gamma_{5}}\left[a_{0} \int \psi_{00}(\xi) \psi_{00}(\xi) \psi_{02}(\xi)+a_{2} \int \psi_{01}(\xi) \psi_{00}(\xi) \psi_{02}(\xi)\right] \\
A_{51}=\frac{1}{\gamma_{5}}\left[a_{0} \int \psi_{00}(\xi) \psi_{10}(\xi) \psi_{02}(\xi)+a_{2} \int \psi_{01}(\xi) \psi_{10}(\xi) \psi_{02}(\xi)\right] \\
A_{52}=\frac{1}{\gamma_{5}}\left[a_{0} \int \psi_{00}(\xi) \psi_{01}(\xi) \psi_{02}(\xi)+a_{2} \int \psi_{01}(\xi) \psi_{01}(\xi) \psi_{02}(\xi)\right] \\
A_{53}=\frac{1}{\gamma_{5}}\left[a_{0} \int \psi_{00}(\xi) \psi_{20}(\xi) \psi_{02}(\xi)+a_{2} \int \psi_{01}(\xi) \psi_{20}(\xi) \psi_{02}(\xi)\right] \\
A_{54}=\frac{1}{\gamma_{5}}\left[a_{0} \int \psi_{00}(\xi) \psi_{11}(\xi) \psi_{02}(\xi)+a_{2} \int \psi_{01}(\xi) \psi_{11}(\xi) \psi_{02}(\xi)\right] \\
A_{55}=\frac{1}{\gamma_{5}}\left[a_{0} \int \psi_{00}(\xi) \psi_{02}(\xi) \psi_{02}(\xi)+a_{2} \int \psi_{01}(\xi) \psi_{02}(\xi) \psi_{02}(\xi)\right]
\end{array}\right.
$$

Here the integral terms corresponding to a zero initial PCE coefficient have been omitted for brevity. Due to the fact that $\boldsymbol{a}_{i}=\left[\mu_{a}, 0, \sigma_{a}, 0, \ldots, 0\right]$, there are only two integral terms in each $\mathbf{A}$ matrix entry. Expressions for higher-order expansions can be derived accordingly to the 1-D and the 2-D case shown here.

\section{References}

${ }^{1}$ Wiener, N., "The Homogeneous Chaos," American Journal of Mathematics, Vol. 60, No. 4, 1938, pp. 897-936.

${ }^{2}$ Ghanem, R. G. and Spanos, P., Stochastic Finite Elements: a Spectral Approach, Springer, New York, USA, 1991.

${ }^{3}$ Xiu, D. and Karniadakis, G. E., "Modeling uncertainty in flow simulations via generalized polynomial chaos," Journal of Computational Physics, Vol. 187, 2003, pp. 137-167.

${ }^{4}$ Xiu, D., Numerical methods for stochastic computations: a spectral method approach, Princeton University Press, New Jersey, USA, 2010.

${ }^{5}$ Xiu, D., "Fast Numerical Methods for Stochastic Computations: A Review," Communications in Computational Physics, Vol. 5, No.2, 2009, pp. 242-272.

${ }^{6}$ Nagy, Z. K. and Braatz, R. D., "Distributional uncertainty analysis using polynomial chaos expansions," 2010 IEEE International Symposium on Computer-Aided Control System Design, IEEE, sep 2010.

${ }^{7}$ Hoover, F. S., "Gradient dynamic optimization with Legendre chaos," Science Direct, Vol. 44, 2008, pp. 135-140.

${ }^{8}$ Prabhakar et al, A., "Polynomial Chaos-Based Analysis of Probabilistic Uncertainty in Hypersonic Flight Dynamics," Journal of Guidance, Control, and Dynamics, Vol. 33, No.1, 2010, pp. 222-234.

${ }^{9}$ Dutta, P. and Bhattachrya, R., "Non Linear Estimation of Hypersonic Flight Using Polynomial Chaos," Journal of Guidance, Control, and Dynamics, Vol. 33, No.6, 2010, pp. 1765-1778.

${ }^{10}$ Fisher, J. and Bhattachrya, R., "Optimal Trajectory Generation with Probabilistic System Uncertainty using Polynomial Chaos," Journal of Dynamic Systems, Measurement and Control, Vol. 133, 2011, pp. 1765-1778.

${ }^{11}$ Xiong, F., Shishi, C., and Xiong, Y., "Dynamic system uncertainty propagation using polynomial chaos," Chinese Journal of Aeronautics, Vol. 27, No.5, 2015, pp. 1156-1170.

${ }^{12}$ Xiong, F., Xiong, Y., and Xue, B., Trajectory Optimization under Uncertainty based on Polynomial Chaos Expansion, Part of AIAA SciTech, AIAA, Kissimmee, Florida USA, 2014.

${ }^{13}$ Ross, I. M., A Primer on Pontryagin's Principle in Optimal Control, Collegiate Publishers, 2015.

${ }^{14}$ Sagliano, M., Development of a Novel Algorithm for High Performance Reentry Guidance, Ph.D. thesis, 2016.

${ }^{15}$ Sagliano, M., Theil, S., Bergsma, M., D'Onofrio, V., Whittle, L., and Viavattene, G., "On the Radau pseudospectral method: theoretical and implementation advances," CEAS Space Journal, Vol. 9, No. 3, Sep 2017, pp. 313-331.

${ }^{16}$ Elissar, "Description of DIDO Optimal Control software," June 2015.

${ }^{17}$ Rao, A. V., Benson, D. A., L., D. C., Patterson, M. A., Francolin, C., I., S., and Huntington, G. T., "GPOPS: A MATLAB Software for Solving Multiple-Phase Optimal Control Problems Using the Gauss Pseudospectral Method," ACM Transactions on Mathematical Software, Vol. 37, No. 2, apr 2010, pp. 1-39.

${ }^{18}$ Gong, Q., Ross, I. M., Kang, W., and Fahroo, F., "Connections Between The Covector Mapping Theorem and Convergence of Pseudospectral Methods for Optimal Control," Comput Optim Appl, 2008, 2008.

${ }^{19}$ Garg, D., Advances in Global Pseudospectral Methods for Optimal Control, Ph.D. thesis, University of Florida, Gainesville, 2011.

${ }^{20}$ Martins, J. R. R., Sturdza, P., and Alonso, J. J., "The Complex-Step Derivative Approximation," ACM Transactions on Mathematical Software, Vol. 29, No. 3, September 2003, Pages 245262, 2003.

${ }^{21}$ Abramovitz, M. and Stegun, I. A., Handbook of Mathematical Functions, Dover Publications, 1695.

${ }^{22}$ Cameron, R. H. and Martin, W. T., "The orthogonal development of non-linear functionals in a series of Fourier-Hermite functionals," Annals of Mathematics, Vol. 48 No. 2, 1947, pp. 385-392.

${ }^{23}$ Askey, R., Orthogonal Polynomials and Special Functions, Society for Industrial and Applied Mathematics, Philadelphia, Pennsylvania, 1975.

${ }^{24}$ Streif, S., Petzke, F., Mesbah, A., Findeisen, R., and Braatz, R. D., Optimal Experimental Design for Probabilistic Model Discrimination Using Polynomial Chaos, 19th IFAC World Congress, IFAC, Cape Town, South Africa, Aug 24-29, 2014. 